\title{
Orbifold equivalence: structure and new examples
}

\author{
Andreas Recknagel Paul Weinreb \\ King's College London, Department of Mathematics, \\ Strand, London WC2R 2LS, UK
}

\begin{abstract}
Orbifold equivalence is a notion of symmetry that does not rely on group actions. Among other applications, it leads to surprising connections between hitherto unrelated singularities. While the concept can be defined in a very general category-theoretic language, we focus on the most explicit setting in terms of matrix factorisations, where orbifold equivalences arise from defects with special properties. Examples are relatively difficult to construct, but we uncover some structural features that distinguish orbifold equivalences most notably a finite perturbation expansion. We use those properties to devise a search algorithm, then present some new examples including Arnold singularities.
\end{abstract}

andreas.recknagel@kcl.ac.uk, paul.weinreb@kcl.ac.uk 


\section{Contents}

1. Introduction 2

2. Orbifold equivalence $\quad 6$

2.1. Definition and general properties . . . . . . . . . . . . . . 6

2.2. Known examples . . . . . . . . . . . . . . . . . . . . . . . 9 9

3. Some structural results on orbifold equivalences 10

3.1. Orbifold equivalences as graded perturbations . . . . . . . . . . . . . . 11

3.2. Weight split criterion . . . . . . . . . . . . . . . 14

4. Algorithmic search, and some concrete results $\quad \mathbf{1 6}$

4.1. Towards an algorithmic search for orbifold equivalences . . . . . . . . . 16

4.2. New examples . . . . . . . . . . . . . . . . . . . . . . . . . . 19

5. Open problems and conjectures 22

A. Appendix: explicit defects

\section{Introduction}

Orbifold equivalence is a phenomenon discovered when trying to describe well-known ideas connected with the action of symmetry groups (originally in quantum field theories) in terms of abstract category-theoretic terms. It turned out that all the data one is interested in when studying these "orbifolds" can be extracted from a separable symmetric Frobenius algebra - which may, but need not, arise from a group action. The abstraction therefore provides a generalised notion of symmetry which does not rely on groups.

The original formulation led to some rather strong results concerning the classification of rational conformal field theories [20], the more abstract bicategory point of view taken in [14] allows for a wider range of applications, including so-called topological LandauGinzburg models.

In the latter context, orbifold equivalence provides a novel equivalence relation for quasi-homogeneous polynomials, leading to unexpected relations between singularities e.g. between simple singularities of types $A$ and $E$ - and to equivalences of categories associated with them, such as categories of matrix factorisations and of representations of quiver path algebras. The new equivalence also implies "dualities" between different topological field theories (correlation functions of one model can be computed in another).

The main aim of the present paper is to construct explicit examples of orbifold equivalences, which in the Landau-Ginzburg context are nothing but matrix factorisations with special properties.

We start by recalling some basic definitions concerning matrix factorisations, then make 
a few remarks about the mathematics and physics context. The "special property" we demand (namely invertible quantum dimensions) will be addressed in section 2. Our main new results will be presented in sections 3 and 4: We uncover some structural properties of orbifold equivalences (they have a finite graded perturbation expansion), exploit this to set up a search algorithm, then list examples of orbifold equivalences found in this way.

A rank $N$ matrix factorisation of a polynomial $W \in \mathbb{C}\left[z_{1}, \ldots, z_{k}\right]$ is a pair of $N \times N$ matrices $E, J$ with polynomial entries satisfying

$$
E J=J E=W \mathbf{1}_{N}
$$

We can collect $E$ and $J$ into a single matrix $Q$,

$$
Q=\left(\begin{array}{cc}
0 & E \\
J & 0
\end{array}\right) \in M_{2 N}(\mathbb{C}[z])
$$

satisfying $Q^{2}=W \mathbf{1}_{2 N}$; we have abbreviated $z=\left(z_{1}, \ldots, z_{k}\right)$. This notation is one way to make the inherent $\mathbb{Z}_{2}$-grading of matrix factorisations explicit: $Q$ anti-commutes with

$$
\sigma=\left(\begin{array}{cc}
\mathbf{1}_{N} & 0 \\
0 & -\mathbf{1}_{N}
\end{array}\right)
$$

and we can use $\sigma$ to decompose the space $M_{2 N}(\mathbb{C}[z])$ into even and odd elements: The former commute with $\sigma$ and are called "bosonic" in the physics context, the latter anticommute with $\sigma$ and are referred to as "fermionic".

Every polynomial $W$ admits matrix factorisations: Any factorisation of $W\left(z_{1}\right)=z_{1}^{p}$ provides a rank 1 factorisation, and once we have matrix factorisations $Q_{a}$ of $W_{a}$ and $Q_{b}$ of $W_{b}$, the so-called tensor product factorisation $Q=Q_{a} \hat{\otimes} Q_{b}$ provides a matrix factorisation of $W=W_{a}+W_{b}$. This $Q$ is formed as in (1.2) from

$$
E:=\left(\begin{array}{cc}
J_{a} \otimes 1 & -1 \otimes E_{b} \\
1 \otimes J_{b} & E_{a} \otimes 1
\end{array}\right), \quad J:=\left(\begin{array}{cc}
E_{a} \otimes 1 & 1 \otimes E_{b} \\
-1 \otimes J_{b} & J_{a} \otimes 1
\end{array}\right)
$$

Unfortunately, it will turn out (see section 3) that matrix factorisations obtained as iterated tensor products of factorisations of monomials of $W$ in general do not have the special additional properties we are interested in.

In the following, we will exclusively focus on graded matrix factorisations:

First of all, we assume that the polynomial $W(z)$ is quasi-homogeneous, i.e. that there exist rational numbers $\left|z_{i}\right|>0$, called the weights of $z_{i}$, such that for any $\lambda \in \mathbb{C}^{\times}:=\mathbb{C} \backslash\{0\}$ we have

$$
W\left(\lambda^{\left|z_{1}\right|} z_{1}, \ldots, \lambda^{\left|z_{k}\right|} z_{k}\right)=\lambda^{D_{W}} W\left(z_{1}, \ldots, z_{k}\right)
$$

for some $D_{W} \in \mathbb{Q}_{+}$, the weight of $W$. Unless specified otherwise, we will assume that $D_{W}=2$, and also that $W \in \mathfrak{m}^{2}$ where $\mathfrak{m}=\left\langle z_{1}, \ldots, z_{k}\right\rangle$ is the maximal ideal of $\mathbb{C}[z]$. For some applications, it is important that the Jacobi $\operatorname{ring} \operatorname{Jac}(W)=\mathbb{C}[z] /\left\langle\partial_{z_{1}} W, \ldots, \partial_{z_{k}} W\right\rangle$ is finite-dimensional as a $\mathbb{C}$-vector space, so let us assume this. 
We will refer to quasi-homogeneous polynomials of weight 2 as "potentials", and to the rational number

$$
\hat{c}(W):=\sum_{i=1}^{k}\left(1-\left|z_{i}\right|\right)
$$

as the central charge of the potential $W$.

In the following, let us use the abbreviation

$$
\lambda \triangleright z:=\left(\lambda^{\left|z_{1}\right|} z_{1}, \ldots, \lambda^{\left|z_{k}\right|} z_{k}\right)
$$

for the $\mathbb{C}^{\times}$-action.

We call a rank $N$ matrix factorisation $Q$ of a potential $W$ graded if there exists a diagonal matrix (the "grading matrix" of $Q$ )

$$
U(\lambda)=\operatorname{diag}\left(\lambda^{g_{1}}, \ldots, \lambda^{g_{2 N}}\right)
$$

with $g_{i} \in \mathbb{Q}$ such that

$$
U(\lambda) Q(\lambda \triangleright z) U(\lambda)^{-1}=\lambda Q(z)
$$

for all $\lambda \in \mathbb{C}^{\times}$. We can set $g_{1}=0$ without loss of generality.

For simplicity, we will assume that $Q(z)$ has no non-zero constant entries. Otherwise $Q$ is decomposable: using row and column transformations, it can be brought into the form $\widetilde{Q} \oplus Q_{\text {triv }}$ where $Q_{\text {triv }}$ is the trivial rank 1 factorisation $W=1 \cdot W$.

Probably the first, very simple, example of a matrix factorisation made its appearance in the Dirac equation, but only with Eisenbud's discovery that free resolutions of modules over $\mathbb{C}[z] /\langle W\rangle$ become periodic [19] was it realised that matrix factorisations can be defined for general potentials and are a useful tools in mathematics.

Later, a category-theoretic point of view was introduced: one can e.g. form a category $\mathrm{hmf}^{\mathrm{gr}}(W)$ whose objects are (finite rank) graded matrix factorisations of $W$, and where (even or odd) morphisms are given by the cohomology of the differential $d_{Q_{1} Q_{2}}$ acting as

$$
d_{Q_{1} Q_{2}}(A)=Q_{1} A-(-1)^{s(A)} A Q_{2}
$$

on $A \in M_{2 N}(\mathbb{C}[z])$, where $s(A)=0$ if $A$ is even with respect to the $\mathbb{Z}_{2}$-grading $\sigma$ in (1.3), and $s(A)=1$ if $A$ is odd.

It was shown that $\mathrm{hmf}^{\mathrm{gr}}(W)$ is equivalent to the derived category of coherent sheaves on zero locus of $W$ - and also to categories of maximal Cohen-Macaulay modules, and of quiver path algebras; see in particular [35], but also [7]. Among these categories, $\mathrm{hmf}^{\mathrm{gr}}(W)$ is the one where explicit computations are easiest to perform.

Let us also make some remarks on the most notable application of matrix factorisations in physics, namely topological Landau-Ginzburg models. (We include these comments mainly because this context is the origin of some of the terminology; an understanding of the physical concepts is not required for the remainder of the paper.) Topological Landau-Ginzburg models are supersymmetric quantum field theories on a two-dimensional 
worldsheet; $W(z)$ appears as interaction potential, the degrees of freedom in the interior of the world-sheet (the "bulk fields") are described by the Jacobi ring $\operatorname{Jac}(W)$.

Within the physics literature, there is strong evidence for a relation between supersymmetric Landau-Ginzburg models with potential $W$ and supersymmetric conformal field theories where a Virasoro algebra with central charge $c=3 \hat{c}(W)$ acts. The conformal field theory is thought to describe the "IR renormalisation group fixed point" of the Landau-Ginzburg model and motivates the term "central charge" for the quantity (1.4).

Additional structure appears if the worldsheet of the Landau-Ginzburg has boundaries: the possible supersymmetry-preserving boundary conditions are precisely the matrix factorisations $Q$ of $W[24,5,26,22]$, and bosonic resp. fermionic degrees of freedom on the boundary are given by the even resp. odd cohomology $H_{Q}^{\bullet}$ of the differential $d_{Q Q}$ defined in eq. (1.7).

Correlation functions in topological Landau-Ginzburg are computed as residues of functions of several complex variables (see e.g. [21] for many details): If the worldsheet has no boundary, the correlator of any element $\phi \in \operatorname{Jac}(W)$ is [39]

$$
\langle\phi\rangle_{W}=\operatorname{res}_{z}\left[\frac{\phi}{\partial_{z_{1}} W \cdots \partial_{z_{k}} W}\right] .
$$

In a model where the worldsheet has a boundary with boundary condition described by a matrix factorisation $Q$ of $W$, one has $[25,22]$

$$
\langle\phi \psi\rangle_{Q}^{\mathrm{KapLi}}=\operatorname{res}_{z}\left[\frac{\phi \operatorname{str}\left(\partial_{z_{1}} Q \cdots \partial_{z_{k}} Q \psi\right)}{\partial_{z_{1}} W \cdots \partial_{z_{k}} W}\right]
$$

for any bulk field $\phi \in \operatorname{Jac}(W)$ and any boundary field $\psi \in H_{Q}^{\bullet}$. The supertrace is defined using the $\mathbb{Z}_{2}$-grading from $(1.3)$, as $\operatorname{str}(A):=\operatorname{tr}(\sigma A)$.

The formula (1.9) is often referred to as Kapustin-Li correlator; a closely related expression will be used, in the next section, to define the "special property" the matrix factorisations of our interest are required to have.

The correlations functions above were first computed in physics, via localisation of path integrals for supersymmetric topological quantum field theories, but they have since been discussed in purely mathematical terms, notably in $[31,17]$.

Instead of worldsheets with boundary, one can also consider worldsheets which are divided into two domains by a "fault line", and the degrees of freedom on the two sides may be governed by two different Landau-Ginzburg potentials $V_{1}(x)$ and $V_{2}(y)$. Such an arrangement is called a (topological) defect, and is described [6] by a matrix factorisation $Q(x, y)$ of $V_{1}(x)-V_{2}(y)$. Degrees of freedom localised on the defect line are described by the morphisms (bosonic or fermionic) of $Q(x, y)$, analogously to the boundary case. Boundary conditions of a Landau-Ginzburg model with potential $V_{1}(x)$ can be viewed as defects between $V_{1}(x)$ and the trivial model $V_{2}=0$.

Topological defects come with additional structure, called the fusion product: In the fault line picture, two defect lines which partition a worldsheet into three regions, with potentials $V_{1}(x), V_{3}\left(x^{\prime}\right)$ in the outer regions and $V_{2}(y)$ in the middle, can be moved 
on top of each other, leaving a single defect between $V_{1}(x)$ and $V_{3}\left(x^{\prime}\right)$. In terms of matrix factorisations, the tensor product $Q_{12}(x, y) \hat{\otimes} Q_{23}\left(y, x^{\prime}\right)$ of two matrix factorisations $Q_{12}(x, y)$ of $V_{1}(x)-V_{2}(y)$ and $Q_{23}\left(y, x^{\prime}\right)$ of $V_{2}(y)-V_{3}\left(x^{\prime}\right)$ is a matrix factorisation of $V_{1}(x)-V_{3}\left(x^{\prime}\right)$. This has infinite rank over $\mathbb{C}\left[x, x^{\prime}\right]$, but is equivalent (by a similarity transformation) to a finite-rank defect [6] depending on $x, x^{\prime}$ only; extracting this finite rank defect yields a representative of the fusion product $Q_{12} \star Q_{23}$. The full construction is somewhat technical (it involves finding and splitting an idempotent morphism of the tensor product, see [10] for details), but implementable on a computer. The construction described in [32] should prove easier to apply.

A concrete mathematical application of defects appeared in the work of Khovanov and Rozansky [27], who proposed to use matrix factorisations to define link invariants that generalise those of Reshetikhin-Turaev ("categorification of the Jones polynomial"). These invariants were made explicitly computable using the fusion product in [10].

At a more abstract level, topological defects in Landau-Ginzburg models, together with structures such as the fusion product, form a bicategory $\mathcal{L G}$, where objects are given by Landau-Ginzburg potentials, 1-morphisms by defects between two potentials (i.e. matrix factorisations of the difference), and 2-morphisms by morphisms (as occurring in (1.7)) of those matrix factorisations. This bicategory is "graded pivotal" $[11,14,8]$, in particular it has adjoints: for each 1-morphism $Q$, i.e. each defect between $V_{1}(x)$ and $V_{2}(y)$, the adjoint $Q^{\dagger}$, a defect between $V_{2}(y)$ and $V_{1}(x)$, is given by

$$
Q^{\dagger}=\left(\begin{array}{cc}
0 & J^{T} \\
-E^{T} & 0
\end{array}\right)
$$

(This equation holds if the number of $y$-variables is even, otherwise there is an additional exchange of $E$ and $J$; see $[11,14,12]$ for details, which will not play a role in what follows.)

A detailed knowledge of category theory is not required to understand the results of the present paper. Indeed, while the category framework is convenient, perhaps even indispensable, to develop the notion of orbifold equivalence and to fully appreciate its wide-ranging applications (including possible extension to higher-dimensional topological field theories), the pedestrian approach via explicit matrix factorisations seems much better suited to construct examples.

\section{Orbifold equivalence}

\subsection{Definition and general properties}

We now come to the definition of the "special property" we require the defects of interest to have. In the following, let $V_{1} \in \mathbb{C}[x]$ and $V_{2} \in \mathbb{C}[y]$, where $x=\left(x_{1}, \ldots, x_{n}\right)$ and $y=\left(y_{1}, \ldots, y_{m}\right)$, be two potentials.

Definition 2.1: $\quad V_{1}$ and $V_{2}$ are orbifold equivalent if there exists a (graded) matrix factorisation $Q(x, y)$ of $V_{1}(x)-V_{2}(y)$ for which the quantum dimensions $q_{L}(Q)$ and $q_{R}(Q)$ are invertible. 
The quantum dimensions of $Q$ are defined as

$$
\begin{aligned}
& q_{L}(Q)=(-1)^{\left(\begin{array}{c}
m+1 \\
2
\end{array}\right)} \operatorname{res}_{x}\left[\frac{\operatorname{str}\left(\partial_{x_{1}} Q \cdots \partial_{x_{n}} Q \cdot \partial_{y_{1}} Q \cdots \partial_{y_{m}} Q\right)}{\partial_{x_{1}} V_{1} \cdots \partial_{x_{n}} V_{1}}\right] \\
& q_{R}(Q)=(-1)^{\left(\begin{array}{c}
n+1 \\
2
\end{array}\right)} \operatorname{res}_{y}\left[\frac{\operatorname{str}\left(\partial_{x_{1}} Q \cdots \partial_{x_{n}} Q \cdot \partial_{y_{1}} Q \cdots \partial_{y_{m}} Q\right)}{\partial_{y_{1}} V_{2} \cdots \partial_{y_{m}} V_{2}}\right]
\end{aligned}
$$

We have used $\operatorname{str}(A)=\operatorname{tr}(\sigma A)$ to abbreviate the supertrace, defined with the help of the

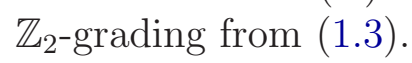

We will call such a $Q$ an orbifold equivalence between $V_{1}$ and $V_{2}$, and we will write $V_{1} \sim_{\text {oeq }} V_{2}$ to indicate that $V_{1}$ and $V_{2}$ are orbifold-equivalent.

We will see below that, in the graded case, the quantum dimensions are complex numbers, so "invertible" simply means "non-zero".

One can show that the quantum dimensions are invariant under permutations of the variables up to a sign [18]. Notice also the close similarity of (2.1) to the Kapustin-Li correlator; this will play a role in section 3 .

One could in principle drop the requirement that $Q$ is a graded matrix factorisation (or even that $V_{1}$ and $V_{2}$ are quasi-homogeneous), the quantum dimensions can be computed for any matrix with polynomial entries. Not much is known in this general situation, so we restrict ourselves to quasi-homogeneous potentials and graded matrix factorisations in this paper.

We summarise some abstract properties of the notions of orbifold equivalence and quantum dimensions in a theorem; all statements were proven before, see $[11,14,13]$ and references therein:

\section{Theorem 2.2:}

(a) $\sim_{\text {oeq }}$ is an equivalence relation.

(b) $V_{1} \sim_{\text {oeq }} V_{1}+y_{1}^{2}+y_{2}^{2}$ (Knörrer periodicity).

(c) If $V_{1}(x) \sim_{\text {oeq }} V_{2}(y)$ and $V_{3}\left(x^{\prime}\right) \sim_{\text {oeq }} V_{4}\left(y^{\prime}\right)$, then $V_{1}(x)+V_{3}\left(x^{\prime}\right) \sim_{\text {oeq }} V_{2}(y)+V_{4}\left(y^{\prime}\right)$. (Note that in this relation each potential depends on a separate set of variables.)

(d) The quantum dimensions do not change under similarity transformations, i.e. $q_{L}(Q)=q_{L}\left(U Q U^{-1}\right)$ for any invertible even matrix $U$; analogously for $q_{R}(Q)$.

(e) The quantum dimensions are additive with respect to forming direct sums: if $Q$ and $\widetilde{Q}$ are two matrix factorisations of $V_{1}-V_{2}$, then $q_{L}(Q \oplus \widetilde{Q})=q_{L}(Q)+q_{L}(\widetilde{Q})$, and analogously for $q_{R}(Q \oplus \widetilde{Q})$.

(f) Up to signs, the quantum dimensions are multiplicative with respect to fusion products $Q \star \widetilde{Q}$, and with respect to forming tensor products $Q_{12}(x, y) \hat{\otimes} Q_{34}\left(x^{\prime}, y^{\prime}\right)$ (where $Q_{12}$ factorises $V_{1}(x)-V_{2}(y)$ and $Q_{34}$ factorises $V_{3}\left(x^{\prime}\right)-V_{4}\left(y^{\prime}\right)$, cf. item c). 
(g) Passing to the adjoint defect interchanges left and right quantum dimensions: $q_{L}\left(Q^{\dagger}\right)=q_{R}(Q)$ and $q_{R}\left(Q^{\dagger}\right)=q_{L}(Q)$.

A quantity of central importance in the bicategory treatment of orbifold equivalences is $A(Q):=Q^{\dagger} \star Q$, sometimes called "symmetry defect". This fusion product is a defect from $V_{2}$ to itself, and it can be shown [14] that

$$
\operatorname{hmf}^{\mathrm{gr}}\left(V_{1}\right) \simeq \bmod \left(Q^{\dagger} \star Q\right)
$$

where the right hand side denotes the category of modules over $A(Q)$, consisting of matrix factorisations of $V_{2}$ on which the defect $A(Q)$ acts via the fusion product. This equivalence of categories is one of several relations existing between structures associated to $V_{1}$ and to $V_{2}$ as soon as the two potentials are orbifold equivalent.

Within the domain of Landau-Ginzburg models, orbifold equivalence leads to a "duality" of the two topological field theories: bulk correlators in the $V_{1}$-model can be computed as correlators in the $V_{2}$-model enriched by defect lines (the defect being $A(Q)$ - see e.g. [14] for a nice pictorial presentation of this fact.

All one needs to prove these statements in the bicategory language is that $A(Q)$ is a "separable symmetric Frobenius algebra". It is this power of abstraction that made it possible to realise that features one is familiar with from orbifold groups can persist without groups being involved.

Explicit computations involving $A(Q)$ can become rather tedious when dealing with complicated orbifold equivalences. However, there is a very simple numerical invariant which contains useful information, namely the (left or right) quantum dimension of $A(Q)$ : Using the facts collected in Theorem 2.2, we find

$$
q_{L}(A(Q))=q_{L}\left(Q^{\dagger}\right) q_{L}(Q)=q_{L}(Q) q_{R}(Q)=q_{R}(A(Q)) .
$$

E.g., if $Q$ is an indecomposable defect with $q_{L}(A(Q)) \neq \pm 1$, then $Q$ is a "true orbifold equivalence" rather than a "mere equivalence" in the bicategory $\mathcal{L G}$, i.e. there cannot be a $\widetilde{Q}$ such that $Q \star \widetilde{Q}$ and $\widetilde{Q} \star Q$ are similar to the unit 1-morphisms $I_{V_{1}}$ and $I_{V_{2}}$ of $V_{1}$ resp. $V_{2}$ (see the next subsection for the definition of $I_{V}$ ).

Perhaps more interestingly, an orbifold equivalence $Q$ does not arise from the action of a finite symmetry group on the potential unless $q_{L}(A(Q))$ is contained in some cyclotomic field: this follows from constraints on the quantum dimensions of orbifold equivalences associated with group actions, see the remarks in the next subsection.

By definition, orbifold equivalence describes a property of a pair of potentials, a defect between them with non-vanishing quantum dimensions merely needs to exist. Ultimately, one would like to be able to read off directly from the potentials whether they are orbifold equivalent or not. So far, however, only the following two facts are known to be necessary criteria for orbifold equivalence:

Proposition 2.3: Using the notations from Def. 2.1, $V_{1}(x)$ and $V_{2}(y)$ are orbifold equivalent only if the total number of variables $n+m$ is even and only if the two potentials have the same central charge, $\hat{c}\left(V_{1}\right)=\hat{c}\left(V_{2}\right)$. 
Both statements were proven in [14], we will give a slightly modified derivation in section 3. The first criterion of Prop. 2.3 is easy to circumvent: if $V_{1}$ and $V_{2}$ have an odd total number of variables, one can pass from $V_{1}$ to $V_{1}+x_{n+1}^{2}$, which has the same central charge. The second criterion, on the other hand, is definitely necessary for the existence of (graded) orbifold equivalences, and a natural question is whether it is already sufficient. We will make a few comments on this in section 5 .

\subsection{Known examples}

We briefly recapitulate the examples of orbifold equivalences known so far.

Reflexivity of $\sim_{\text {oeq }}$ is ensured by the identity defect, an orbifold equivalence $I_{V}(x, y)$ between $V(x)$ and $V(y)$ with quantum dimensions $q_{L}\left(I_{V}\right)=q_{R}\left(I_{V}\right)=1$. This takes the form of a nested tensor product $I_{V}:=Q_{(1)} \hat{\otimes} \cdots \hat{\otimes} Q_{(n)}$ of rank 1 matrix factorisations with

$$
E_{(i)}=\left[V\left(x_{1}, \ldots, x_{i}, y_{i+1}, \ldots y_{n}\right)-V\left(x_{1}, \ldots, x_{i-1}, y_{i}, \ldots, y_{n}\right)\right] /\left(x_{i}-y_{i}\right), \quad J_{(i)}=x_{i}-y_{i} .
$$

In the bicategory picture, the identity defect provides the unit (with respect to the fusion product) 1-morphism in $\operatorname{End}(V)$. In the Landau-Ginzburg context, $I_{V}$ can be thought of as an "invisible defect".

The example from which "orbifold equivalences" derive their name involves a symmetry group $G$ of the potential $V$, i.e. a finite subgroup of $\mathbb{C}[x]$-automorphisms which leaves $V$ invariant. Then one can, for each $g \in G$, construct "twisted" identity defects $I_{V}^{g}$ formed like $I_{V}$ above, with $J_{i}$ replaced by $J_{i}^{g}=x_{i}-g\left(y_{i}\right)$, and $E_{i}$ replaced accordingly. Details are given in $[6,14,3,4]$, where it is also shown that the quantum dimensions of $I_{V}^{g}$ are given by $\operatorname{det}(g)^{ \pm 1}$ (hence contained in the cyclotomic field determined by the order of the symmetry group $G$ ). In this special situation, the symmetry defect $A(Q)$ from above is given by the separable symmetric Frobenius algebra $A(Q)=\bigoplus_{g \in G} I_{V}^{g}$, from which one can extract complete information about the orbifolded topological Landau-Ginzburg model.

The most interesting orbifold equivalences so far have been found for simple singularities of ADE type $[14,13]$. The potentials are

$$
V_{A_{n}}=x_{1}^{n+1}+x_{2}^{2}, \quad V_{D_{d}}=x_{1}^{d-1}+x_{1} x_{2}^{2}, \quad V_{E_{6}}=x_{1}^{3}+x_{2}^{4}, \quad V_{E_{7}}=x_{1}^{3}+x_{1} x_{2}^{3}, \quad V_{E_{8}}=x_{1}^{3}+x_{2}^{5}
$$

with $d \geq 2$ and $n \geq 4$, the corresponding Landau-Ginzburg models are related to socalled $N=2$ superconformal minimal models with central charge $\hat{c}<1$. It turns out that whenever two of these potentials have the same central charge, they are also orbifold equivalent; the classes with more than one representative are $\left\{A_{d-1}, D_{d / 2+1}\right\}$ for even $d$ not equal to 12,18 or 30 , and $\left\{A_{11}, D_{7}, E_{6}\right\},\left\{A_{17}, D_{10}, E_{7}\right\}$ and $\left\{A_{29}, D_{16}, E_{8}\right\}$. The $A$ - $D$ orbifold equivalences are related to (simple current) orbifolds in the CFT context, but the $A-E$ orbifold equivalences do not arise from any group action [13]; they are examples of "symmetries" beyond groups.

For the purposes of elucidating some general observations to be made later, and also of conveying an idea of the typical complexity of the matrix factorisations involved, we 
reproduce a concrete example of an orbifold equivalence from [13], namely that between $V_{A_{11}}=x_{1}^{12}+x_{2}^{2}$ and $V_{E_{6}}=y_{1}^{3}+y_{2}^{4}$. In this case, the smallest possible (see subsection 3.2) orbifold equivalence is of rank 2. With $Q$ formed from $E$ and $J$ as in (1.2), the matrix elements of $E$ are given by

$$
\begin{aligned}
& E_{11}=y_{2}^{2}-x_{2}+\frac{1}{2} y_{1}\left(s x_{1}\right)^{2}+\frac{2 t+1}{8}\left(s x_{1}\right)^{6} \\
& E_{12}=-y_{1}+y_{2}\left(s x_{1}\right)+\frac{t+1}{4}\left(s x_{1}\right)^{4} \\
& E_{21}=y_{1}^{2}+y_{1} y_{2}\left(s x_{1}\right)+\frac{t}{4} y_{1}\left(s x_{1}\right)^{4}+\frac{2 t+1}{4} y_{2}\left(s x_{1}\right)^{5}-\frac{9 t+5}{48}\left(s x_{1}\right)^{8} \\
& E_{22}=y_{2}^{2}+x_{2}+\frac{1}{2} y_{1}\left(s x_{1}\right)^{2}+\frac{2 t+1}{8}\left(s x_{1}\right)^{6}
\end{aligned}
$$

$J=-\operatorname{adjugate}(E)$, and the complex coefficients $s, t$ satisfy the algebraic equations $t^{2}=1 / 3, \quad s^{12}=-576(26 t-15)$. This defect has non-zero quantum dimensions, namely $q_{L}(Q)=s, q_{R}(Q)=3(1-t) / s$.

In [33], an explicit rank 4 orbifold equivalence was written down between two of the fourteen (quasi-homogeneous) exceptional unimodal Arnold singularities (list e.g. in [1]), namely between $E_{14}$ and $Q_{10}$ described by the potentials

$$
V_{E_{14}}(x)=x_{1}^{8}+x_{2}^{2}+x_{3}^{3} \text { and } V_{Q_{10}}(y)=y_{1}^{4}+y_{1} y_{2}^{2}+y_{3}^{3}
$$

both having central charge $\hat{c}=\frac{13}{12}$. However, one should notice that this orbifold equivalence already follows from the $A-D$ results of [14] and the general property Theorem 2.2 (c): One can write $E_{14}=A_{7} \times A_{2}$ and $Q_{10}=D_{5} \times A_{2}$, and one has $A_{7} \sim_{\text {oeq }} D_{5}$. In the same way, one can of course construct other orbifold equivalences at arbitrarily high central charge, simply by "adding up" suitable simple singularities with $\hat{c}<1$.

\section{Some structural results on orbifold equivalences}

If one tries to generate examples of orbifold equivalences truly beyond simple singularities, one soon realises that the approach taken in [13] is neither general nor systematic enough. In that work, the method employed to find expressions like (2.4) was to set one of the variables $x_{i}, y_{j}$ occurring in $W(x, y)=V_{1}(x)-V_{2}(y)$ to zero, to pick some simple matrix factorisation $\widetilde{Q}$ of the resulting potential $\tilde{W}$ and to complete $\widetilde{Q}$ to a graded matrix factorisation $Q(x, y)$ of the full $W(x, y)$ using quasi-homogeneous entries that contain the missing variable - under additional simplifying constraints such as $J=-\operatorname{adjugate}(E)$. But as soon as one has to cope with a larger number of variables, or higher rank, one needs a lot of luck to hit a good starting point $\widetilde{Q}$.

Nevertheless, the computations in [13] contain germs of ideas which can be formulated in general terms and exploited in a systematic manner. In this section, we will show that every graded orbifold equivalence has a (finite) perturbation expansion, a structure from which one can draw some general conclusion on the form $Q(x, y)$ must take. The grading is a crucial ingredient, and we will present a constraint on the allowed grading matrices in subsection 3.2. Together with the perturbative structure, this will enable us to devise a relatively efficient search algorithm for orbifold equivalences in section 4 . 


\subsection{Orbifold equivalences as graded perturbations}

Given a matrix factorisation $Q$ of a potential $W$, one can ask whether $Q$, or the differential $d_{Q Q}$ associated with it, admits deformations. As is familiar in the context of deformation theory, deformation directions are controlled by $\mathrm{Ext}^{1}$ - or $H_{Q}^{1}$, the space of boundary fermions -, obstructions by $\mathrm{Ext}^{2}-$ or $H_{Q}^{0}$. References and some results can e.g. be found in $[9]$.

We will now show that graded orbifold equivalences, or indeed any graded defect between $V_{1}(x)$ and $V_{2}(y)$, can be naturally viewed as a deformation of a matrix factorisation of $V_{1}(x)$, with the variables $y_{j}$ featuring as deformation parameters and $-V_{2}(x) \mathbf{1}_{2 N}$ as obstruction term. Since we are not restricting to the vanishing locus of the obstruction term, we speak of "perturbations" rather than deformations.

That the defect is graded has a very desirable consequence: the perturbation expansion terminates after finitely many steps.

For this discussion, it is convenient to introduce some further notions concerning graded matrix factorisations: Let $U(\lambda)$ be a grading matrix as in (1.6). Borrowing some further physics terminology, we say that a matrix $A \in M_{2 N}(\mathbb{C}[z])$ has "R-charge" $R$ wrt. the grading $U(\lambda)$ if

$$
U(\lambda) A(\lambda \triangleright z) U(\lambda)^{-1}=\lambda^{R} A(z) .
$$

(The graded matrix factorisation $Q$ in (1.6) then has R-charge 1.)

This relation implies that the entries $A_{r s}$ of $A$ are quasi-homogeneous polynomials in the $z_{i}$. Their weights can be computed from the grading matrix as

$$
w\left(A_{r s}\right)=g_{s}-g_{r}+R \text { for } r, s=1, \ldots, 2 N .
$$

In the special case $A=Q$ we will sometimes write $w(Q)$ for the matrix formed from the $w\left(Q_{r s}\right)$ and call it the weight matrix of $Q$; analogously we will use $w(E), w(J)$ for the weight matrices of $E$ and $J$ related to $Q$ as in (1.2).

By way of a brief excursion, and also as a step towards a proof of Prop. 2.3, let us use the notion of R-charges to provide a self-contained derivation of a statement that is wellknown in the physics literature on topological Landau Ginzburg models, namely that the correlators $(1.8,1.9)$ have a "background charge". Instead of employing arguments from an underlying twisted conformal field theory, this can be derived from properties of the residue. We focus on the Kapustin-Li correlator here:

Proposition 3.1: $\quad$ Set $z=\left(z_{1}, \ldots, z_{k}\right)$ and let $Q(z)$ be a (graded) rank $N$ matrix factorisation of a potential $W(z)$ with $\operatorname{dim}_{\mathbb{C}}(\operatorname{Jac}(W))<\infty$. Let $\psi \in M_{2 N}(\mathbb{C}[z])$ be a morphism of definite $\mathbb{Z}_{2}$-degree $s(\psi)$ and definite $\mathrm{R}$-charge $R_{\psi}$. Then

$$
\langle\psi\rangle_{Q}^{\mathrm{KapLi}}=0
$$

unless $s(\psi)+k$ is even and unless $R_{\psi}=\hat{c}(W)$.

Proof: The statement on the $\mathbb{Z}_{2}$-degree follows because $Q$ and its partial derivatives are odd matrices wrt. to the $\mathbb{Z}_{2}$-grading $\sigma$, hence a product of $n$ of these with the even or odd matrix $\psi$ has no diagonal terms, hence zero supertrace, if $k+s(\psi)$ is odd. 
As the Jacobi ring of $W$ is a finite-dimensional $\mathbb{C}$-vector space, for each $i=1, \ldots, k$ there is a $\nu_{i} \in \mathbb{Z}_{+}$and polynomials $C_{i j}$ such that $z_{i}^{\nu_{i}}=\sum_{j} C_{i j}(z) \partial_{z_{j}} W(z)$. This implies, see e.g. [21], that

$$
\operatorname{res}_{z}\left[\frac{f}{\partial_{z_{1}} W \cdots \partial_{z_{k}} W}\right]=\operatorname{res}_{z}\left[\frac{\operatorname{det}(C) f}{z_{1}^{\nu_{1}} \cdots z_{k}^{\nu_{k}}}\right]
$$

for any polynomial $f(z)$.

In the case at hand, $f=\operatorname{str}\left(\partial_{z_{1}} Q \cdots \partial_{z_{k}} Q \cdot \psi\right)$, and since $Q$, its derivatives, and $\psi$ have definite $\mathrm{R}$-charges, a rescaling of the $z_{i}$ can be traded for conjugation with the grading matrix $U(\lambda)$ - this leaves the supertrace invariant - up to extra prefactors $\lambda^{1-\left|z_{i}\right|}$ resp. $\lambda^{r \psi}$ from relation (3.1). Hence $f$ is quasi-homogeneous of weight $R_{\psi}+\hat{c}(W)$.

It is easy to see that $\operatorname{det}(C)$ is quasi-homogeneous of weight $-k-\hat{c}(W)+\sum_{i} \nu_{i}\left|z_{i}\right|$. The residue projects $f \cdot \operatorname{det}(C)$ onto the monomial $z_{1}^{\nu_{1}-1} \cdots z_{k}^{\nu_{k}-1}$, which has weight $-k+$ $\hat{c}(W)+\sum_{i} \nu_{i}\left|z_{i}\right|$. Thus the residue can be non-zero only if $R_{\psi}=\hat{c}(W)$.

Turning to the perturbation expansion of orbifold equivalences, we assume, as before, that $V_{1}(x)$ and $V_{2}(y)$ are quasi-homogeneous potentials of weight 2, without linear terms, and we denote the weights of the variables by $\left|x_{i}\right|$ for $i=1, \ldots, n$ resp. $\left|y_{j}\right|$ for $j=1, \ldots, m$. We abbreviate $W(x, y):=V_{1}(x)-V_{2}(y)$.

Proposition 3.2: Assume that $Q(x, y)$ is a (graded) rank $N$ orbifold equivalence between $V_{1}(x)$ and $V_{2}(y)$, i.e. $Q^{2}=W \mathbf{1}_{2 N}$ and $q_{L}(Q) q_{R}(Q) \neq 0$. Set $Q_{1}(x):=\left.Q(x, y)\right|_{y=0}$ and $F_{j}:=\left.\partial_{y_{j}} Q(x, y)\right|_{y=0}$ for $j=1, \ldots, m$. Then

(1) $\quad F_{j}$ is a fermionic morphism of $Q_{1}$ with R-charge $R_{j}=1-\left|y_{j}\right|$, for all $j=1, \ldots, m$.

(2) The left quantum dimension of $Q$ can be written as

$$
q_{L}(Q)=\left\langle F_{1} \cdots F_{m}\right\rangle_{Q_{1}}^{\mathrm{KapLi}}
$$

where $\langle\cdots\rangle_{Q_{1}}^{\mathrm{KapLi}}$ denotes the Kapustin-Li boundary correlator of the LG model with bulk potential $V_{1}$ and boundary condition $Q_{1}$.

(3) $Q(x, y)$ has a finite perturbation expansion, with $y_{j}$ appearing as parameters:

$$
Q(x, y)=\sum_{\kappa=0}^{\kappa_{\max }} Q^{(\kappa)}(x, y) \quad \text { with } Q^{(0)}(x, y)=Q_{1}(x) \text { and } Q^{(1)}(x, y)=\sum_{j} y_{j} F_{j} .
$$

The higher order terms satisfy

$$
\left\{Q_{1}, Q^{(\kappa)}\right\}+\sum_{\lambda=1}^{\kappa-1} Q^{(\kappa-\lambda)} Q^{(\lambda)}=-V_{2}^{(\kappa)}
$$

where $\{\cdot, \cdot\}$ denotes the anti-commutator and where $V_{2}^{(\kappa)}$ is the order $\kappa$ term of $V_{2}$. 
Proof: The $F_{j}$ are odd wrt. the $\sigma$-grading (as $Q$ is), and they are in the kernel of $d_{Q_{1} Q_{1}}$ because $\left\{Q_{1}, F_{j}\right\}=\left.\partial_{y_{j}} Q(x, y)^{2}\right|_{y=0}=\left.\partial_{y_{j}} W \mathbf{1}\right|_{y=0}=0$. Let $U(\lambda)$ be the grading for $Q(x, y)$ - and, for that matter, for $Q_{1}(x)$. Differentiating (1.6) gives

$$
U(\lambda) \partial_{y_{j}} Q(\lambda \triangleright x, \lambda \triangleright y) U(\lambda)^{-1}=\lambda^{1-\left|y_{j}\right|} \partial_{y_{j}} Q(x, y)
$$

so in particular the $F_{j}$ are fermions of $Q_{1}$ with definite R-charge $R_{j}=1-\left|y_{j}\right|$.

To see the second statement, note that the left quantum dimension of a graded matrix factorisation is a quasi-homogeneous polynomial in $y$, and in fact has to be a (non-zero) number in order to be invertible. Hence $q_{L}(Q)$ does not depend on the $y$-variables. Setting $y=0$ in the first of the residue formulas (2.1) directly produces the Kapustin-Li correlator (1.9) of the product $F_{1} \cdots F_{m}$ of "boundary fermions" in the $\left(V_{1}, Q_{1}\right)$ theory.

Eq. (3.3) simply follows from a Taylor expansion of $Q^{2}=W \mathbf{1}_{2 N}$ around $y=0$, keeping in mind that $Q_{1}(x)$ is a matrix factorisation of $V_{1}(x)$.

Finiteness of the perturbation series can be seen by analysing R-charges and weight matrices: The order $\kappa$ term $Q^{(\kappa)}(x, y)=\sum_{\vec{p}} M_{\vec{p}}^{(\kappa)} y^{\vec{p}}$ is a linear combination of monomials in the $y_{j}, \vec{p} \in \mathbb{Z}_{+}^{m}$ with $p_{1}+\ldots+p_{m}=\kappa$, with matrix-valued coefficients $M_{\vec{p}}^{(\kappa)} \in M_{2 N}(\mathbb{C}[x])$. These coefficients are odd wrt. the $\mathbb{Z}_{2}$-grading, and they have R-charge $R_{\vec{p}}^{(\kappa)}=1-p_{1}\left|y_{1}\right|-$ $\ldots-p_{m}\left|y_{m}\right|$. The entries of $M_{\vec{p}}^{(\kappa)}$ are homogeneous polynomials in the $x_{i}$, the weight of the $r$-s-entry is $w_{r s}=g_{s}-g_{r}+R_{\vec{p}}^{(\kappa)}$, where the $g_{r}$ define the grading matrix $U(\lambda)$ as before. Since all the variable weights are strictly positive, for large enough $\kappa$ not only the $\mathrm{R}$-charge but also the weights $w_{r s}$ will become negative for all $r, s \in\{1, \ldots, 2 N\}$, which implies that $M_{\vec{p}}^{(\kappa)}$ has to vanish.

An analogous expansion can be performed around $x=0$, and the right quantum dimension of $Q(x, y)$ takes the form of a correlator of boundary fermions in the LandauGinzburg with bulk potential $-V_{2}(y)$ and boundary condition $\left.Q_{2}(y)\right):=\left.Q(x, y)\right|_{x=0}$. Setting $\tilde{F}_{i}:=\left.\partial_{x_{i}} Q(x, y)\right|_{x=0}$, we have

$$
q_{R}(Q)=\left\langle\tilde{F}_{1} \cdots \tilde{F}_{n}\right\rangle_{Q_{2}}^{\mathrm{KapLi}}
$$

In the present paper, the main application of Prop. 3.2 will be to devise a more systematic search algorithm for orbifold equivalences, which allows us to tackle more difficult situations than the simple singularities discussed in [13]. But there are some immediate structural consequences implied by the perturbation expansion:

First off, the condition $\hat{c}\left(V_{1}\right)=\hat{c}\left(V_{2}\right)$ necessary for the existence of a graded orbifold equivalence follows immediately from the "background charge" of topological LandauGinzburg correlators: The product $F_{1} \cdots F_{m}$ is a morphism with R-charge $\sum_{j}\left(1-\left|y_{j}\right|\right)=$ $\hat{c}\left(V_{2}\right)$, and its Kapustin-Li correlator in the $\left(V_{1}, Q_{1}\right)$-model vanishes, according to the statement rederived in Prop. 3.1, unless this R-charge coincides with the background charge $\left.\hat{c}\left(V_{1}\right)\right)$ of that model.

Prop. 3.2 also constrains what form the matrix elements of an orbifold equivalence $Q(x, y)$ can take: Clearly, for each $j=1, \ldots, m$ there must be a $Q$-entry that contains a term linear in $y_{j}$, lest one of the partial derivatives $F_{j}$ is zero; likewise for the $x_{i}$. 
(In fact, none of the $F_{j}$ can be trivial in the $Q_{1}$-cohomology, i.e. none can be of the form $F_{j}=\left[Q_{1}, A_{j}\right]$ for some $A_{j}$, because the Kapustin-Li form is independent of the representative of the cohomology class.)

Moreover, under very mild additional assumptions on the potentials, one can show that orbifold equivalences must involve some "entanglement" of the $x$ - and $y$-variables:

Proposition 3.3: Assume that $V_{2}(y) \in \mathfrak{m}^{3}$, i.e. has no quadratic or lower order terms. Then an orbifold equivalence $Q(x, y)$ between $V_{1}(x)$ and $V_{2}(y)$ must have mixed $x y$-terms, i.e. it cannot have the form $Q(x, y)=Q_{1}(x)+Q_{2}(y)$.

Proof: Assume $Q(x, y)=Q_{1}(x)+Q_{2}(y)$. The first summand is a matrix factorisation of $V_{1}(x)$, the second summand one of $-V_{2}(y)$; consequently $\left\{Q_{1}(x), Q_{2}(y)\right\}=0$ and also $\left\{\partial_{x_{i}} Q_{1}, F_{j}\right\}=0$. Moreover,

$$
0=-\left.\partial_{y_{j_{1}}} \partial_{y_{j_{2}}} V_{2}(y)\right|_{y=0}=\left\{F_{j_{1}}, F_{j_{2}}\right\}+\left.\left\{Q_{2}(y), \partial_{y_{j_{1}}} \partial_{y_{j_{2}}} Q_{2}(y)\right\}\right|_{y=0}
$$

The last term vanishes (since the matrix factorisations we consider have no constant terms), so all the $F_{j}$ anti-commute and square to zero.

Let $N:=\sigma \partial_{x_{1}} Q_{1} \cdots \partial_{x_{n}} Q_{1} F_{1} \cdots F_{m}$, which is the argument of the trace in the residue formula for $q_{L}(Q)$. In the case at hand, this matrix $N$ is nilpotent,

$$
N^{2}= \pm\left(\partial_{x_{1}} Q_{1} \cdots \partial_{x_{n}} Q_{1}\right)^{2}\left(F_{1} \cdots F_{m}\right)^{2}=0
$$

hence $\operatorname{tr}(N)=0$ and $q_{L}(Q)=0$.

Note that we can relax the assumption on $V_{2}(y)$ : as soon as there is one variable $y_{j *}$ such that $\left.\partial_{y_{j^{*}}} \partial_{y_{j}} V_{2}(y)\right|_{y=0}=0$ for all $j=1, \ldots, m$, we have that $F_{j^{*}}$ anti-commutes with all $F_{j}$, and $N$ is nilpotent.

In particular, this result rules out the simplest tensor products as orbifold equivalences (under the stated assumptions on the potentials): if $Q(x, y)=Q_{a}(x) \hat{\otimes} Q_{b}(y)$ where $Q_{a}(x)$ is a matrix factorisation of $V_{1}(x)$ and $Q_{b}(y)$ one of $-V_{2}(y)$, then $Q(x, y)$ has zero quantum dimensions.

That the standard method (forming tensor products) of constructing matrix factorisations for complicated polynomials is barred when seeking orbifold equivalences goes some way in explaining why the latter are hard to find. Results of the type of Prop. 3.3 may also prove useful for showing that equality of central charges is an insufficient criterion for two potentials to be orbifold equivalent.

\subsection{Weight split criterion}

The perturbative expansion described in Prop. 3.2 is a useful ingredient of an algorithmic search for orbifold equivalences, but, as it stands, the need to select a grading and a $Q_{1}(x)$ as starting point seems to limit efficiency quite severely. In this subjection, we will point out that the gradings (i.e. the weight matrices of $Q_{1}(x, y)$ and $Q(x, y)$ ) are subject to a highly selective criterion - a criterion that applies to any graded matrix factorisation $Q(z)$ of any quasi-homogeneous potential $W(z)$, not just to defects. 
It will be more convenient to rescale the variable weights such that all $\left|z_{i}\right|$ are natural numbers; so for the time being, the weight of $W(z)$ is given by some integer $D_{W} \in \mathbb{Z}_{+}$, not necessarily equal to 2 .

Before giving a general formulation, let us see the criterion "at work" in the concrete example of the $A_{11}-E_{6}$ orbifold equivalence found in [13] and reproduced in subsection 2.2. Here, $V_{A_{11}}(x)=x_{1}^{12}+x_{2}^{2}, V_{E_{6}}(y)=y_{1}^{3}+y_{2}^{4}$, and $W(z)=V_{A_{11}}(x)-V_{E_{6}}(y)$ with $z=(x, y)$. The variable weights are $\left|x_{1}\right|=1,\left|x_{2}\right|=6,\left|y_{1}\right|=4,\left|y_{2}\right|=3$ (after scaling up to integers, so that $D_{W}=12$.)

Any graded matrix factorisation $E J=J E=W \mathbf{1}_{N}$ must in particular contain (quasihomogeneous) polynomials factorising the $x_{2}^{2}$-term from $W$ - and such factors must occur in each row and each column of $E$ and $J$. Up to constant prefactors, these polynomials must be of the form $x_{2}+f_{r s}$ for some $f_{r s}$ having the same weight as $x_{2}$. So each row and each column of the weight matrices $w(E)$ and $w(J)$ must contain a 6 .

Likewise, the $y_{1}^{3}$-term has to be factorised, so each row and column of $w(E)$ and $w(J)$ has to contain a 4 (from a factor $y_{1}^{1}+\ldots$ ) or an 8 (from a factor $y_{1}^{2}+\ldots$ ).

If we want to construct a rank $N=2$ matrix factorisation of $W=V_{A_{11}}-V_{E_{6}}$, these two observations (together with the constraint that $Q$ should be graded) fix the weight matrices completely, up to row and column permutations and up to swapping $E$ and $J$ :

$$
w(E)=\left(\begin{array}{ll}
6 & 4 \\
8 & 6
\end{array}\right)
$$

which is indeed the weight matrix for the $A_{11}-E_{6}$ orbifold equivalence (2.4) found by Carqueville et al. (Thanks to the low rank and the small number of variables, it is fairly easy to arrive at a concrete $Q$ once the above $w(E)$ is known.)

In order to formulate the criterion in general, we need some notation. Let

$$
W(z)=\sum_{\tau=1}^{T} m_{\nu}(z)
$$

be the decomposition of the potential into monomial terms; each $m_{\tau}$ has weight $D_{W}$. For each $\tau=1, \ldots, T$, let $S_{\tau}$ be the set of weights of possible non-trivial divisors of $m_{\tau}$, i.e.

$$
S_{\tau}=\left\{w \in\left\{1, \ldots, D_{W}-1\right\}: \exists f \in \mathbb{C}[z] \text { s.th. } f \text { divides } m_{\tau} \text { and } f \text { has weight } w\right\}
$$

Weight split criterion: If $Q(z)$ is a graded matrix factorisation of $W(z)$ with weight matrix $w(Q)$, then each row and and column of $w(Q)$ contains an element of $S_{\tau}$ for all $\tau=1, \ldots, T$.

Let us look at two further examples to illustrate the usefulness of this criterion. For the two unimodal Arnold singularities $V_{E_{13}}(x)=x_{2}^{3}+x_{2} x_{1}^{5}$ and $V_{Z_{11}}(y)=y_{1}^{3} y_{2}+y_{2}^{5}$, the variable weights are $\left|x_{1}\right|=2,\left|x_{2}\right|=5,\left|y_{1}\right|=4,\left|y_{2}\right|=3$ (re-scaled so that $W$ has weight 15). The terms in $W$ admit weight splits $5+10$ and $5+10=7+8=9+6=11+4=13+2$ (from $E_{13}$ ) and $12+3=8+7=4+11$ and $3+12=6+9$ (from $Z_{11}$ ). In each row 
and each column of $w(E)$, there must be a 5 or a 10 , and there must be one from the set $\{3,4,7,8,11,12\}$.

One can just about fit the above weights into a rank 2 matrix $w(E)$ with entries $5,12,10,3$, but this leads to zero quantum dimensions (the associated $Q$ are tensor products and ruled out as orbifold equivalences by Prop. 3.3).

At rank 3, one can form 24 weight matrices $w(E)$ satisfying the weight split criterion, and one of those leads to an orbifold equivalence, see the next section. It is worth mentioning that the "successful" $w(E)$ is one where many entries are members of both the weight split lists $S_{\tau}$ coming from $E_{13}$ and the weight split lists coming from $\left(Z_{11}\right)$; these offer the best opportunity for an "entanglement" of $x$ and $y$ variables.

How restrictive the weight split criterion can be is demonstrated when one tries to construct an orbifold equivalence for the Arnold singularities $Z_{13}$ and $Q_{11}$, see section 4: here, one needs a rank 6 matrix factorisation, and of about 2.7 million conceivable weight matrices $w(Q)$ only 60 pass the criterion.

There are additional restrictions on viable weight matrices $w(Q)$ which apply if $Q$ is to be an orbifold equivalence between $V_{1}(x)$ and $V_{2}(y)$. E.g., the requirement that non-trivial fermions of given R-charge have to exist (needed for non-zero quantum dimensions, cf. Prop 3.2), means that for each variable $y_{j}$, at least one of the $w(Q)$-entries must be of the form $\left|y_{j}\right|+n(x)$ where $n(x)$ is some $\mathbb{Z}_{+}$-linear combination of the weights $\left|x_{i}\right|$; analogously with the roles of $x$ and $y$ interchanged. In the examples we studied, this condition from existence of fermions turned out to be far less restrictive than the weight split criterion arising from the matrix factorisation conditions.

\section{Algorithmic search, and some concrete results}

In this chapter, we will present some new examples of orbifold equivalences. Most of them were discovered using an algorithm based on the perturbative expansion introduced in the previous section. First, we make some general remarks on the "computability" of orbifold equivalences and outline a computer-implementable algorithm to deal with the problem, then we list the new examples themselves.

\subsection{Towards an algorithmic search for orbifold equivalences}

The question whether there is a rank $N$ orbifold equivalence $Q$ between two given potentials $V_{1}$ and $V_{2}$ can be converted into an ideal membership problem and, for fixed $N$, can be decided by a finite computation.

To see this, let us write the matrix elements of $Q$ as

$$
Q_{r s}=\sum_{\vec{p}} a_{r s, \vec{p}} z^{\vec{p}} \quad \text { for } \quad r, s \in\{1, \ldots, 2 N\}
$$

where $z=\left(x_{1}, \ldots, x_{n}, y_{1}, \ldots, y_{m}\right)$ and where $\vec{p} \in \mathbb{Z}_{+}^{m+n}$ is a multi-index. The main "trick" now is to shift one's focus away from the variables $z$ and work in a ring of polynomials in the $a_{r s, \vec{p}}$ : 
The requirement that $Q$ is a rank $N$ matrix factorisation of $W(z)=V_{1}(x)-V_{2}(y)$ imposes polynomial (in fact: bilinear) equations $f_{\alpha}^{\mathrm{MF}}(a)=0$ on the coefficients $a_{r s, \vec{p}} \in \mathbb{C}$. ( $\alpha$ labels the various bilinear equations, $a$ collectively denotes all the coefficients.)

The quantum dimensions can be computed, using definition (2.1), whether or not $Q$ is a matrix factorisation; for a graded $Q$, one obtains two polynomials (of degree $n+m$ ) in the $a_{r s, \vec{p}}$. The requirement that both quantum dimensions are non-zero is equivalent to the single equation

$$
f^{\mathrm{qd}}(a):=q_{L}(Q) q_{R}(Q) a_{\text {aux }}-1=0
$$

being solvable, where $a_{\text {aux }}$ is an additional auxiliary coefficient.

Thus, the matrix $Q$ is an orbifold equivalence between $V_{1}$ and $V_{2}$ if and only if the system

$$
f_{\alpha}^{\mathrm{MF}}=0, \quad f^{\mathrm{qd}}=0
$$

of polynomial equations in the coefficients $a_{r s, \vec{p}}$ and $a_{\text {aux }}$ has a solution. By Hilbert's weak Nullstellensatz, this is the case iff

$$
1 \notin\left\langle f_{\alpha}^{\mathrm{MF}}, f^{\mathrm{qd}}\right\rangle_{\mathbb{C}[a]}
$$

This type of ideal membership problem can be tackled rather efficiently with computer algebra systems like Singular. (Such systems are usually restricted to working over $\mathbb{Q}$, but for potentials $V_{1}, V_{2}$ with rational coefficients it is enough to study (4.3) over the rationals in order to prove or disprove existence of an orbifold equivalence with coefficients $a_{r s, \vec{p}}$ in the algebraic closure $\overline{\mathbb{Q}}$.)

Once a grading $U(\lambda)$, hence a weight matrix for $Q$, has been chosen, it is easy to write down the most general homogeneous matrix elements $Q_{r s}$ (4.1) that conform with this grading. Moreover, there is only a finite number of possible gradings $U(\lambda)=\operatorname{diag}\left(\lambda^{g_{1}}, \ldots, \lambda^{g_{2 N}}\right)$ for a given rank $N$. To see this, recall that the weights of the $Q$-entries are given by $w\left(Q_{r s}\right)=g_{s}-g_{r}+1$ (we set the weight of the potential to 2), and also that we can fix $g_{1}=0$ wlog - so in particular $w\left(Q_{1 r}\right)=g_{r}+1$ and $w\left(Q_{r 1}\right)=-g_{r}+1$. Therefore, at least one of the $g_{r}$ has to satisfy $-1 \leq g_{r} \leq 1$, otherwise the entire first row or column of $Q$ would have to vanish (because the weights would all be negative), which would contradict the matrix factorisation conditions. We can repeat the argument for the $g_{r}$ nearest to $g_{1}$ and find, overall, that $g_{r} \in[-2 N, 2 N]$ for all $r$. Finally, $Q_{r s}$ can be a non-zero polynomial in the $x_{i}, y_{j}$ only if its weight $w\left(Q_{r s}\right)$ is a sum of the (finitely many, rational) weights $\left|x_{i}\right|,\left|y_{j}\right|$, hence only finitely many choices $g_{r}$ from the interval $[-2 N, 2 N]$ can lead to a graded rank $N$ matrix factorisation of $V_{1}(x)-V_{2}(y)$.

All in all, the question whether there exists a rank $N$ orbifold equivalence between two given potentials $V_{1}, V_{2}$ can be settled in principle. Our guess is that there is an upper bound $N_{\max }\left(V_{1}, V_{2}\right)$ such that, if no orbifold equivalence of rank $N<N_{\max }\left(V_{1}, V_{2}\right)$ exists, then none exists at all - but we have only circumstantial evidence: all known (indecomposable) examples of orbifold equivalences have rank smaller than the nested tensor product matrix factorisation obtained by factorising each monomial in $V_{1}-V_{2}$; and packing a matrix factorisation "too loosely" risks making the supertrace inside the quantum dimensions vanish. 
So much for the abstract question whether orbifold equivalence is a property that can be decided algorithmically at all. In order to search for concrete examples, we have devised an algorithm based on the perturbation expansion and the weight split criterion introduced in section 3 :

(a) From the potentials $V_{1}(x), V_{2}(y)$, compute the variable weights $\left|x_{i}\right|,\left|y_{j}\right|$.

(b) Choose a rank $N$.

(c) Exploiting the weight split criterion from subsection 3.2, compute all admissible gradings (i.e. weight matrices) for this rank.

(d) Choose a weight matrix and form the most general matrix factorisation $Q_{1}(x)$ of $V_{1}(x)$ with this weight matrix.

(e) For each $y_{j}$, compute the space of fermions $F_{j}$ of $Q_{1}(x)$ with R-charges $1-\left|y_{j}\right|$.

(f) For any R-charge $R_{M}$ that can occur in the expansion of $Q(x, y)$ from Prop. 3.2, determine the space of odd matrices with that R-charge.

(g) Compute $Q(x, y)$ using the conditions from Prop. 3.2 (c), then compute the quantum dimensions $q_{L}(Q)$ and $q_{R}(Q)$. (Everything will depend on unknown coefficients $a$.)

(h) Extract the conditions $f_{\alpha}^{\mathrm{MF}}(a)=0$ and $f^{\mathrm{qd}}(a)=0$ on the coefficients appearing in $Q(x, y)$ and check whether this system of polynomial equations admits a solution.

Computer algebra systems such as Singular have in-built routines to perform the last step, employing (variants of) Buchberger's algorithm to compute a Gröbner basis of the ideal spanned by $f^{\mathrm{MF}}, f^{\mathrm{qd}}$.

Already when forming $Q_{1}(x)$ with a given weight matrix, undetermined coefficients $a$ enter the game - but far fewer than would show up in the most general matrix $Q(x, y)$ with the same weight matrix, because one only uses the $x$-variables to form quasi-homogeneous entries: the perturbation expansion from Prop. 3.2 "organises" the computation to some extent from the outset. Nevertheless, even for harmless looking potentials $V_{1}, V_{2}$ one can easily end up with close to one thousand polynomial equations in hundreds of unknowns $a_{r s, \vec{p}}$. Due to restrictions on memory and run-time, it is advisable in practice to make guesses for some of the coefficients $a_{r s, \vec{p}}$ occurring in $Q(x, y)$ or already in $Q_{1}(x)$, instead of trying to tackle the most general ansatz. We have succeeded in automatising most of the steps involved in making the equations tractable for Singular, some of the results are collected in the next subsection.

Finding an explicit solution for the coefficients $a$ is of course desirable, but not necessary to prove that two potentials are orbifold equivalent. It appears that Singular is not the optimal package for determining explicit solutions (although it is very efficient in establishing solvability); feeding the polynomial equations resulting from the Singular code into Mathematica, say, might be more promising. 
If one is content with existence statements, additional avenues are open: One could e.g. employ numerical methods to find approximate solutions to the system of equations (4.2), then check whether any of them satisfies the criteria of the Kantorovich theorem or of Smale's $\alpha$-theory. If so, one has proven (rigorously) that there is an exact solution in a neighbourhood of the numerical one. We did not take this route, but it might lead to a more efficient computational tool towards a classification of orbifold equivalent potentials.

\subsection{New examples}

We now present new examples of orbifold equivalences, starting with a few isolated (but hard-won) cases, including all remaining pairs of unimodal Arnold singularities, then adding a series of equivalences obtained by simple transformations of variables.

Theorem 4.1: In each of the following cases, the potential $V_{1}(x)$ is orbifold equivalent to the potential $V_{2}(y)$ :

(1) $V_{1}(x)=x_{1}^{6}+x_{2}^{2}$ and $V_{2}(y)=y_{1}^{3}+y_{2}^{3}$.

(These are the singularities $A_{5}$ resp. $A_{2} \times A_{2}$, at central charge $\hat{c}=\frac{2}{3}$.)

(2) $V_{1}(x)=x_{1}^{5} x_{2}+x_{2}^{3}$ and $V_{2}(y)=y_{1}^{3} y_{2}+y_{2}^{5}$.

(These are two of the exceptional unimodal Arnold singularities, namely $E_{13}$ resp. $Z_{11}$, at central charge $\hat{c}=\frac{16}{15}$.)

(3) $V_{1}(x)=x_{1}^{6}+x_{1} x_{2}^{3}+x_{3}^{2}$ and $V_{2}(y)=y_{2} y_{3}^{3}+y_{2}^{3}+y_{1}^{2} y_{3}$.

(These are the exceptional unimodal Arnold singularities $Z_{13}$ resp. $Q_{11}$, at central charge $\hat{c}=\frac{10}{9}$.)

(4) $V_{1}(x)=x_{1}^{2} x_{3}+x_{2} x_{3}^{2}+x_{2}^{4}$ and $V_{2}(y)=-y_{1}^{2}+y_{2}^{4}+y_{2} y_{3}^{4}$.

(These are the exceptional unimodal Arnold singularities $S_{11}$ resp. $W_{13}$, at central charge $\hat{c}=\frac{9}{8}$.)

(5) $V_{1}(x)=x_{1}^{10} x_{2}+x_{2}^{3}$ and $V_{2}(y)=y_{1} y_{2}^{7}+y_{1}^{3} y_{2}$.

(These are a chain resp. a loop (or cycle), in the nomenclature of [29, 23], at central charge $\hat{c}=\frac{6}{5}$, a value shared by the pair $Q_{17}$ and $W_{17}$ of bimodal Arnold singularities.)

Proof: In contrast to $E_{14}-Q_{10}$, none of these cases can be traced back to known results on simple singularities. Lacking, therefore, any elegant abstract arguments, we can only establish these orbifold equivalences by finding explicit matrix factorisations $Q$ of $V_{1}-V_{2}$ with non-zero quantum dimensions. The ranks of the $Q$ we found are, in the order of the cases in the theorem, 2, 3, 6, 4 and 3. In most cases, $Q$ depends on coefficients $a$ which are subject to (solvable!) systems of polynomial equations. We list those matrices on the web-page [36], in the form of a Singular-executable text file. This page also provides a few small Singular routines to perform the necessary checks: extraction of 
the matrix factorisation conditions (bilinear equations on the $a$ ), computation of the quantum dimensions, computation of the Gröbner basis for the ideal in (4.3). For the sake of completeness, and in order to give an impression of the complexity, the matrices and the polynomial equations are also reproduced in the appendix of the present paper.

In all of the five cases, the orbifold equivalence satisfies $q_{L}(Q) q_{R}(Q) \neq \pm 1$, hence $A=Q^{\dagger} \star Q$ is not similar to the identity defect: these are "true orbifold equivalences", not "mere equivalences in the bicategory $\mathcal{L} \mathcal{G}$ ".

The web-page mentioned above also presents direct orbifold equivalences between $D_{7}$ and $E_{6}$, between $D_{10}$ and $E_{7}$, and between $D_{16}$ and $E_{8}$. That these simple singularities are orbifold equivalent follows already from the $A-D$ and $A-E$ results in $[14,13]$, what makes the direct $D-E$ defects noteworthy is that they have at most rank 3. (The smallest orbifold equivalence between $E_{8}$ and $A_{29}$ is of rank 4.)

Together with the straightforward $E_{14}-Q_{10}$ orbifold equivalence mentioned in section 2, Theorem 4.1 exhausts all orbifold equivalences among the (quasi-homogeneous) exceptional unimodal Arnold singularities: no other pairs with equal central charge exist among those fourteen potentials. The orbifold equivalent pairs are precisely the pairs that display "strange duality" (Dolgachev and Gabrielov numbers are interchanged), see e.g. [38].

Among the 14 exceptional bimodal Arnold singularities, only $Q_{17}$ and $W_{17}$ have the same central charge (namely $\hat{c}=\frac{6}{5}$.); we have not yet found an orbifold equivalence between them (nor between $Q_{17}$ or $W_{17}$ and the pair in item (5) above).

It might be worth mentioning that the arguments one can use to treat the $E_{14}-Q_{10}$ case - i.e. Theorem 2.2 (c) - also show that orbifold equivalence does not respect the modality of a singularity:

The exceptional unimodal Arnold singularity $Q_{12}$ with $V_{Q_{12}}(x)=x_{1}^{5}+x_{1} x_{2}^{2}+x_{3}^{3}$ is orbifold equivalent to the exceptional bimodal Arnold singularity $E_{18}$ given by $V_{E_{18}}(y)=y_{1}^{10}+y_{2}^{3}+$ $y_{3}^{2}$ : the former is $D_{6} \times A_{2}$, the latter $A_{9} \times A_{2}$, and $D_{6} \sim_{\text {oeq }} A_{9}$ due to the results of [14]. By the same method, one can relate other exceptional Arnold singularities to sums of simple singularities; among the examples involving bimodal singularities are $Q_{16} \sim_{\text {oeq }} A_{13} \times A_{2}$ and $U_{16} \sim_{\text {oeq }} E_{8} \times A_{2} \sim_{\text {oeq }} A_{5} \times A_{4}$.

A number of more or less expected orbifold equivalences, including infinite series, can be established via transformations of variables:

Lemma 4.2: Assume $Q(x, y)$ is an orbifold equivalence between $V_{1}(x)$ and $V_{2}(y)$, and assume that $y \mapsto y^{\prime}$ is an invertible, weight-preserving transformation of variables. Then $Q\left(x, y^{\prime}\right)$ is an orbifold equivalence between $V_{1}(x)$ and $V_{2}\left(y^{\prime}\right)$ if the weights $\left|y_{i}\right|$ are pairwise different, or if $V_{2}(y) \in \mathfrak{m}^{3}$.

Proof: First, focus on the variable transformation itself: We can assume wlog. that the $y_{1}, \ldots, y_{m}$ are labeled by increasing weight, $y_{1}$ having the lowest weight. Then the transformation can be written as $y_{j} \mapsto y_{j}^{\prime}=f_{j}(y)+\sum_{k \in I_{j}} A_{j k} y_{k}$ where $A_{j k} \in \mathbb{C}$, where $I_{j}=\left\{k:\left|y_{k}\right|=\left|y_{j}\right|\right\}$ and where $f_{j}$ depends only on those $y_{l}$ with $\left|y_{l}\right|<\left|y_{j}\right|$. As $y \mapsto y^{\prime}$ 
preserves weights, $f_{j}$ has no linear terms. The Jacobian $\mathcal{J}$ of the transformation is lower block-diagonal and $\operatorname{det}(\mathcal{J})=\operatorname{det}(A)$, a non-zero constant.

Since $Q^{\prime}:=Q\left(x, y^{\prime}\right)$ is obviously a matrix factorisation of $V_{1}(x)-V_{2}\left(y^{\prime}\right)$, we only need to study the quantum dimensions of $Q^{\prime}$. The relation $q_{R}\left(Q^{\prime}\right)=\operatorname{det}(A) q_{R}(Q)$ results immediately from making a substitution of integration variables in the formula for the right quantum dimension.

The left quantum dimension of $Q^{\prime}$ can be expressed as a Kapustin-Li correlator (in the $\left(V_{1}, Q_{1}\right)$ model) of the fermions $F_{j}^{\prime}=\left.\partial_{y_{j}^{\prime}} Q^{\prime}\right|_{y^{\prime}=0}=\left.\sum_{l=1}^{m} \frac{\partial y_{l}}{\partial y^{\prime} j}\right|_{y=0} F_{l}$. Here, we have already exploited $y^{\prime}=0 \Leftrightarrow y=0$ to simplify, but the summation over $l$ might still lead to linear combinations which are difficult to control. The extra assumptions on $V_{2}(y)$ avoid this: If all $\left|y_{j}\right|$ are pairwise different, then $\left.\frac{\partial y_{l}}{\partial y^{\prime} j}\right|_{y=0}=b_{j} \delta_{j, l}$ for some non-zero constants $b_{j}$. If $V_{2}$ starts at order 3 or higher, the $F_{j}$ anti-commute with each other inside the correlator: adapting the proof of Prop. 3.3, one finds

$$
0=-\left.\partial_{y_{j_{1}}} \partial_{y_{j_{2}}} V_{2}\right|_{y=0}=\left\{F_{j_{1}}, F_{j_{2}}\right\}+\left\{Q_{1}(x),\left.\partial_{y_{j_{1}}} \partial_{y_{j_{2}}} Q(x, y)\right|_{y=0}\right\}
$$

and the last term vanishes in the $Q_{1}$-cohomology, therefore does not contribute to the Kapustin-Li correlator. Hence, the correlator is totally anti-symmetric in the $F_{j}$, and the linear combination of correlators making up the left quantum dimension is simply $q_{L}\left(Q^{\prime}\right)=\operatorname{det}(A)^{-1} q_{L}(Q)$.

Applying this lemma to the identity defect of $V_{1}(x)-V_{1}(y)$, one can establish orbifold equivalences e.g. in the following cases:

(1) So-called "auto-equivalences" of unimodal Arnold singularities: different descriptions of the same singularity exist for $U_{12}, Q_{12}, W_{12}, W_{13}, Z_{13}$ and $E_{14}$. The assumptions on the variable weights resp. structure of $V_{2}$ made in Lemma 4.2 hold for all these cases. These orbifold equivalences were already discussed in [34], and although the concrete formulas given there contain errors, the general structure $(Q$ being a nested tensor product of rank 4) coincides with what one obtains from the identity defect upon a weight-preserving transformation of variables.

The auto-equivalence between $V_{Q_{17}^{T}}(x)=x_{1}^{3} x_{2}+x_{2}^{5} x_{3}+x_{3}^{2}$ and $V(y)=y_{1}^{3} y_{2}+y_{2}^{10}+y_{3}^{2}$ is another such example, involving a bimodal Arnold singularity.

(2) Equivalences between quasi-homogeneous polynomials of Fermat, chain and loop (or cycle) type at $\hat{c}<1$ :

$$
\begin{aligned}
& V_{A_{2 n-1}}(x)=x_{1}^{2 n}+x_{2}^{2} \text { and } V_{D_{n+1}^{T}}(y)=y_{1}^{n} y_{2}+y_{2}^{2} \\
& V_{L_{n}}(x)=x_{1}^{n} x_{2}+x_{1} x_{2}^{2} \text { and } V_{D_{2 n}}(y)=y_{1}^{2 n-1}+y_{1} y_{2}^{2} \\
& V_{C_{n}}(x)=x_{1}^{2} x_{2}+x_{2}^{n} x_{3}+x_{3}^{2} \text { and } V_{D_{2 n+1}}(y)=y_{1}^{2 n}+y_{1} y_{2}^{2}+y_{3}^{2}
\end{aligned}
$$

with $n \geq 2$ in all three pairs. Explicit orbifold equivalences for $A-D^{T}$ were already given in [37].

(3) Cases involving non-trivial marginal bulk deformations, e.g. 
at central charge $\hat{c}=\frac{10}{9}$, one finds an orbifold equivalence between the product $A_{8} \times A_{2}$ of simple singularities, $V_{\left(A_{8} \times A_{2}\right)}(x)=x_{1}^{9}+x_{2}^{3}$, and special deformations of $Z_{13}^{T}$, given by $V_{Z_{13}^{T}}(y)=y_{1}^{6} y_{2}+y_{2}^{3}+\mu_{2} y_{1}^{3} y_{2}^{2}$, if $\mu_{2}= \pm \sqrt{3}$;

at central charge $\hat{c}=\frac{8}{7}$, the two deformed singularities $V_{E_{19}^{T}}(x)=x_{1}^{3} x_{2}+x_{2}^{7}+$ $\mu_{1} x_{1} x_{2}^{5}$ and $V_{2}(y)=y_{1} y_{2}^{5}+y_{1}^{3} y_{2}+\mu_{2} y_{1}^{2} y_{2}^{3}$ are orbifold equivalent as long as the two deformation parameters are related by $\mu_{1}=\mu\left(\frac{1}{3} \mu_{2}^{2}-1\right)$ with $3 \mu^{3}=-\mu_{2}\left(\frac{2}{9} \mu_{2}^{2}-1\right)$.

Lemma 4.2 can also be used to prove an orbifold equivalence one would expect on geometric grounds: The elliptic curve is described by $V_{\lambda}(x)=-x_{2}^{2} x_{3}+x_{1}\left(x_{1}-x_{3}\right)\left(x_{1}-\right.$ $\left.\lambda x_{3}\right)$, where $\lambda$ is a complex parameter with $\lambda \neq 0,1$, and two such curves $V_{\lambda}$ and $V_{\lambda^{\prime}}$ describe birationally equivalent tori if and only if

$$
\lambda^{\prime} \in\{\lambda, 1-\lambda, 1 / \lambda, 1 /(1-\lambda),(\lambda-1) / \lambda, \lambda /(\lambda-1)\} .
$$

One can apply a weight-preserving variable transformation to bring $V_{\lambda}$ into an alternative form $V_{e}(y)=-y_{2}^{2} y_{3}+\left(y_{1}-e_{1} y_{3}\right)\left(y_{1}-e_{2} y_{3}\right)\left(y_{1}-e_{3} y_{3}\right)$. The parameters of the two forms are related by $\lambda=\left(e_{3}-e_{1}\right) /\left(e_{2}-e_{1}\right)$, and the six different $\lambda^{\prime}$-values in (4.4) arise from permuting the $e_{i}$, which of course leaves $V_{e}$ unchanged; thus we find $V_{\lambda^{\prime}} \sim_{\text {oeq }} V_{\lambda}$.

Since, in all the examples listed after Lemma 4.2, we start from the identity defect, the orbifold equivalence resulting from the transformation of variables automatically satisfies $q_{L}\left(Q^{\prime}\right) q_{R}\left(Q^{\prime}\right)=1$, so it is likely that they are "mere equivalences" in the bicategory $\mathcal{L} \mathcal{G}$. (One way to verify this would be to compute and analyse the fusion product $\left(Q^{\prime}\right)^{\dagger} \star Q^{\prime}$.) But Lemma 4.2 could also be applied to the orbifold equivalence between $D_{n+1}$ and $A_{2 n-1}$, say, to produce a defect with $q_{L}\left(Q^{\prime}\right) q_{R}\left(Q^{\prime}\right)=2$ between $D_{n+1}$ and $D_{n+1}^{T}$.

Furthermore, the potentials of type $D_{n}^{T}, C_{n}$ and $L_{n}$ listed in item (2) appear as separate entries in lists of quasi-homogeneous polynomials [29, 23], but not in lists of singularities (where more general types of transformations of variables are allowed to identify two singularities). The orbifold equivalences given in item (2) of Lemma 4.2 may not be surprising, but it is not clear to us whether there are abstract theorems guaranteeing that polynomials which are equivalent as singularities are (orbifold) equivalent in $\mathcal{L G}$.

A first edition of an "oeq catalogue", i.e. a list of polynomials sorted into orbifold equivalence classes based on the results of $[14,13]$ and our new findings, is available at the web-page [36].

\section{Open problems and conjectures}

Ultimately, one would like to find a simple (combinatorial or number-theoretic) criterion that allows to read off directly from the potentials $V_{1}, V_{2}$ whether they are orbifold equivalent or not - instead of taking a detour via constructing an explicit orbifold equivalence $Q$.

Having invested quite a lot of effort into finding such matrices $Q$, the authors sincerely hope that such a criterion involves conditions beyond the ones listed in Prop. 2.3. 
And there are indeed reasons to believe that $\hat{c}\left(V_{1}\right)=\hat{c}\left(V_{2}\right)$ alone is insufficient for $V_{1} \sim_{\text {oeq }} V_{2}$ :

One line of arguments concerns marginal deformations: Le $V$ be a potential which admits a marginal deformation, i.e. there is a quasi-homogeneous element $\phi \in \operatorname{Jac}(V)$ of weight 2. (The Fermat elliptic curve $V(x)=\sum_{i=1}^{3} x_{i}^{3}$ with $\phi=x_{1} x_{2} x_{3}$ is an example.) Set $V_{1}(x)=V(x)+\mu \phi(x)$, where $\mu \in \mathbb{C}$ is a deformation parameter, and $V_{2}(y)=V(y)$; we have $\hat{c}\left(V_{1}\right)=\hat{c}\left(V_{2}\right)$.

The examples of orbifold equivalences listed at the end of section 4, involving $Z_{13}^{T}$, $E_{19}^{T}$ and the geometrically equivalent tori (4.4), already suggest that a given method of constructing a defect $Q$ between $V+\mu \phi$ and $V$ might lead to an orbifold equivalence for a discrete set of $\mu$-values only.

In general, let $Q(x, y ; \mu)$ be rank $N$ matrix factorisation of $V_{1}(x)-V_{2}(y)$ and assume its $\mu$-derivative exists in a neighbourhood of $\mu=0$. Then the bosonic morphism $\Phi:=$ $\phi(x) \mathbf{1}_{2 N}=\left\{Q, \partial_{\mu} Q\right\}$ is zero in the cohomology of $Q$, and $\mathbf{1} \otimes \Phi$ is zero in the cohomology of $Q^{\dagger} \hat{\otimes} Q$. This should imply that $\Phi$ is absent from $\operatorname{End}(A)$ for $A=Q^{\dagger} \star Q$, which in turn makes it unlikely that there is a projection from $\operatorname{End}(A)$ to $\operatorname{Jac}(V)$ - but the latter has to be the case [14] if $Q$ is an orbifold equivalence.

A more direct proof that $c\left(V_{1}\right)=\hat{c}\left(V_{2}\right)$ does not guarantee orbifold equivalence might result from incompatibility of the "weight split lists" $S_{\tau}$ occurring in the weight split criterion from subsection 3.2.

We conjecture that orbifold equivalences $Q$ have trivial fermionic cohomology.

This is true in every concrete case for which we have computed $H_{Q}^{1}$, and the conjecture is backed up by the following observation: If a matrix factorisation $Q$ of $W$ has a non-trivial fermion $\psi \in H_{Q}^{1}$, one can form the cone

$$
C_{\psi}(\lambda)=\left(\begin{array}{cc}
Q & \lambda \psi \\
0 & Q
\end{array}\right)
$$

which is again a matrix factorisation of $W$ for any $\lambda \in \mathbb{C}$. The upper triangular form implies that $q_{L}\left(C_{\psi}(\lambda)\right)=q_{L}(Q \oplus Q)=2 q_{L}(Q)$ for any value of $\lambda$ (likewise for the right quantum dimension). In general, however, cones $C_{\psi}(\lambda)$ with $\lambda \neq 0$ are not equivalent (related by similarity transformations) to the direct sum $Q \oplus Q$, so one would not expect the quantum dimensions to always coincide.

A related question (related due to the role of fermions in deformations of matrix factorisations) is whether there can be moduli spaces of orbifold equivalences between two fixed potentials, or whether the equations only ever admit a discrete set of solutions. Our computations point towards the latter, but we have no proof.

The bicategory setting might provide a better language in which to tackle these general questions.

We hope that the orbifold equivalences presented here prove fruitful in singularity theory, and in other areas related to matrix factorisations by well-established equivalences of categories, but one should also explore applications of orbifold equivalence in string theory, or in the context of mirror symmetry. 
E.g., orbifold equivalences between Arnold singularities may also imply relations between $N=2$ supersymmetric gauge theories in 4 dimensions "engineered" from these singularities, see $[15,16]$.

It is reasonable to expect that potentials related by "Berglund-Hübsch-Krawitz" duality $[2,28]$ are orbifold equivalent $\left(E_{13} \sim_{\text {oeq }} Z_{11}\right.$ is one example), and this duality is one approach to constructing mirror manifolds.

One might also explore whether some of the orbifold equivalences of Landau-Ginzburg potentials can be "lifted" to relations of the conformal field theories associated with them. In particular, the question whether there is a CFT analogue to $A_{5} \sim_{\text {oeq }} A_{2} \times A_{2}$, perhaps in terms of an orbifold construction, should be accessible because the central charge is that of a theory of two free bosons. The $A_{2}$-model is assocatiated with a free boson compactified on a circle, see [30] and references therein; we do not know whether a similar statement can be made for $A_{5}$.

Let us add some speculative comments on orbifold equivalence and entanglement. In quantum physics, entanglement refers to the phenomenon that a physical system comprised from two subsystems (like an electron-positron pair) can be in a state such that observations made on one subsystem immediately determine properties of the second subsystem no matter how great the separation between the two. This behaviour has no analogue in classical physics.

Already the general consequences implied by an orbifold equivalence $V_{1} \sim_{\text {oeq }} V_{2}-$ e.g. the relation (2.2) between categories, or more directly the one between correlators in the Landau-Ginzburg models associated with $V_{1}(x)$ and $V_{2}(y)$ - are strongly reminiscent of entanglement.

Closer to the level of concrete formulas, one notices that quantum states displaying entanglement are formed from states describing the subsystems in a manner that resembles the mixing of $x$ - and $y$-variables implied by Prop. 3.3.

Indeed, we expect that the quantum dimensions of a defect can be related to a suitably defined entanglement entropy in Landau-Ginzburg models.

If this can be made manifest and the "symmetries" discussed here can ultimately be traced back to quantum entanglement, perhaps "entanglement equivalence" might be a more appropriate term than "orbifold equivalence".

\section{Acknowledgements}

We are indebted to N. Carqueville for introducing us to the problem, for many valuable discussions, and for an early version of the Singular code to compute quantum dimensions. We also thank I. Brunner, D. Murfet and I. Runkel for useful conversations and comments.

The work of P.W. was partially supported by an STFC studentship. 


\section{A. Appendix: explicit defects}

For the sake of completeness, we collect the orbifold equivalences that can serve to prove Theorem 4.1. The Singular-executable formats given on the web-page [36] should be of more practical use.

To save writing zeroes, we list matrices $E$ and $J$ only. $Q$ is constructed from them as in (1.2). For fear of producing typos, we have largely refrained from attempts at simplifying the Singular output (except for the very easy case $A_{5} \sim_{\text {oeq }} A_{2} \times A_{2}$ ). The matrices spelled out in the following are the simplest ones we could find: what results from our Singular algorithm typically contains many more coefficients $a_{r s, \vec{p}}$, and we have chosen explicit values for some of them.

The orbifold equivalences are listed in the order they appear in Theorem 4.1.

(1) A rank 2 orbifold equivalence between $A_{5}$ and $A_{2} \times A_{2}$ :

$$
\begin{gathered}
E=\left(\begin{array}{cc}
x_{1}^{2}-a_{1}\left(y_{1}+y_{2}\right) & x_{2}+a_{2} x_{1}\left(y_{1}-y_{2}\right) \\
x_{2}-a_{2} x_{1}\left(y_{1}-y_{2}\right) & -x_{1}^{4}-64 a_{1}^{8} y_{2}^{2}+16 a_{1}^{5} y_{1} y_{2}-a_{1} x_{1}^{2}\left(y_{1}+y_{2}\right)-4 a_{1}^{2} y_{1}^{2}
\end{array}\right) \\
J=\left(\begin{array}{cc}
x_{1}^{4}+64 a_{1}^{8} y_{2}^{2}-16 a_{1}^{5} y_{1} y_{2}+a_{1} x_{1}^{2}\left(y_{1}+y_{2}\right)+4 a_{1}^{2} y_{1}^{2} & x_{2}+a_{2} x_{1}\left(y_{1}-y_{2}\right) \\
x_{2}-a_{2} x_{1}\left(y_{1}-y_{2}\right) & -x_{1}^{2}+a_{1}\left(y_{1}+y_{2}\right)
\end{array}\right)
\end{gathered}
$$

where the coefficients have to satisfy

$$
a_{2}^{2}=3 a_{1}^{2} \quad \text { and } \quad a_{1}^{3}=\frac{1}{4} .
$$

The quantum dimensions of $Q$ are $q_{L}(Q)=-2 a_{1} a_{2}$ and $q_{R}(Q)=-\frac{4}{3} a_{2}$. Since their product is 2 , this is a "true orbifold equivalence", not an ordinary equivalence in the bi-category of Landau-Ginzburg potentials. On the other hand, since 2 is contained in any cyclotomic field, a group action might be the source of this orbifold equivalence.

(2) An ugly rank 3 orbifold equivalence between $E_{13}$ and $Z_{11}$ :

The matrix elements $E_{r s}$ and $J_{r s}$ are given by

$$
\begin{aligned}
& E_{11}=-x_{1}^{2}-y_{1} a_{2} \\
& E_{12}=-x_{1} y_{2} a_{3}-x_{1} y_{2} a_{4}+x_{2} \\
& E_{13}=-y_{2} a_{4} \\
& E_{21}=x_{1} y_{2} a_{3}-x_{1} y_{2} a_{5}+x_{2} \\
& E_{22}=y_{2}^{2} a_{1}^{2} a_{4}^{2}+y_{2}^{2} a_{1} a_{3} a_{4}+y_{2}^{2} a_{1} a_{4}^{2}+y_{2}^{2} a_{1} a_{4} a_{5}-x_{1}^{3} a_{1}+y_{2}^{2} a_{3}^{2}+y_{2}^{2} a_{3} a_{4}-y_{2}^{2} a_{3} a_{5}+y_{2}^{2} a_{5}^{2}+x_{1} y_{1} a_{1} a_{2}- \\
& x_{1} y_{1} a_{1} a_{6}+x_{1}^{3}-y_{2}^{2} a_{7} \\
& E_{23}=x_{1}^{2}+y_{1} a_{6} \\
& E_{31}=-x_{1}^{3} a_{1}-x_{1}^{3}-y_{2}^{2} a_{7} \\
& E_{32}=-x_{1}^{2} y_{2} a_{1}^{2} a_{4}+2 y_{1} y_{2} a_{1}^{2} a_{4} a_{2}-x_{1}^{2} y_{2} a_{1} a_{3}-x_{1}^{2} y_{2} a_{1} a_{4}-x_{1}^{2} y_{2} a_{1} a_{5}+y_{1} y_{2} a_{1} a_{3} a_{2}+2 y_{1} y_{2} a_{1} a_{4} a_{2}+ \\
& y_{1} y_{2} a_{1} a_{5} a_{2}-y_{1} y_{2} a_{1} a_{4} a_{6}-x_{1}^{2} y_{2} a_{3}+x_{1}^{2} y_{2} a_{5}+y_{1} y_{2} a_{3} a_{2}-y_{1} y_{2} a_{4} a_{6}-y_{1} y_{2} a_{5} a_{6}+x_{1} x_{2} \\
& E_{33}=x_{1} y_{2} a_{5}+x_{2} \\
& J_{11}=-x_{1} y_{2}^{3} a_{1}^{2} a_{4}^{2} a_{5}-x_{1}^{4} y_{2} a_{1}^{2} a_{4}-x_{1} y_{2}^{3} a_{1} a_{3} a_{4} a_{5}-x_{1} y_{2}^{3} a_{1} a_{4}^{2} a_{5}-x_{1} y_{2}^{3} a_{1} a_{4} a_{5}^{2}+2 x_{1}^{2} y_{1} y_{2} a_{1}^{2} a_{4} a_{2}- \\
& x_{1}^{2} y_{1} y_{2} a_{1}^{2} a_{4} a_{6}+2 y_{1}^{2} y_{2} a_{1}^{2} a_{4} a_{2} a_{6}-x_{1}^{4} y_{2} a_{1} a_{3}-x_{1}^{4} y_{2} a_{1} a_{4}-x_{2} y_{2}^{2} a_{1}^{2} a_{4}^{2}-x_{1} y_{2}^{3} a_{3}^{2} a_{5}-x_{1} y_{2}^{3} a_{3} a_{4} a_{5}+
\end{aligned}
$$


$x_{1} y_{2}^{3} a_{3} a_{5}^{2}-x_{1} y_{2}^{3} a_{5}^{3}+x_{1}^{2} y_{1} y_{2} a_{1} a_{3} a_{2}+2 x_{1}^{2} y_{1} y_{2} a_{1} a_{4} a_{2}-x_{1}^{2} y_{1} y_{2} a_{1} a_{3} a_{6}-2 x_{1}^{2} y_{1} y_{2} a_{1} a_{4} a_{6}+y_{1}^{2} y_{2} a_{1} a_{3} a_{2} a_{6}+$ $2 y_{1}^{2} y_{2} a_{1} a_{4} a_{2} a_{6}+y_{1}^{2} y_{2} a_{1} a_{5} a_{2} a_{6}-y_{1}^{2} y_{2} a_{1} a_{4} a_{6}^{2}-x_{1}^{4} y_{2} a_{3}-x_{2} y_{2}^{2} a_{1} a_{3} a_{4}-x_{2} y_{2}^{2} a_{1} a_{4}^{2}-x_{2} y_{2}^{2} a_{1} a_{4} a_{5}+$ $x_{1}^{2} y_{1} y_{2} a_{3} a_{2}-x_{1}^{2} y_{1} y_{2} a_{3} a_{6}-x_{1}^{2} y_{1} y_{2} a_{4} a_{6}+y_{1}^{2} y_{2} a_{3} a_{2} a_{6}-y_{1}^{2} y_{2} a_{4} a_{6}^{2}-y_{1}^{2} y_{2} a_{5} a_{6}^{2}+x_{1} y_{2}^{3} a_{5} a_{7}+x_{1}^{3} x_{2} a_{1}-$ $x_{2} y_{2}^{2} a_{3}^{2}-x_{2} y_{2}^{2} a_{3} a_{4}+x_{2} y_{2}^{2} a_{3} a_{5}-x_{2} y_{2}^{2} a_{5}^{2}-x_{1} x_{2} y_{1} a_{1} a_{2}+x_{1} x_{2} y_{1} a_{1} a_{6}+x_{1} x_{2} y_{1} a_{6}+x_{2} y_{2}^{2} a_{7}$ $J_{12}=-x_{1}^{2} y_{2}^{2} a_{1}^{2} a_{4}^{2}-y_{1} y_{2}^{2} a_{1}^{2} a_{4}^{2} a_{2}-x_{1}^{2} y_{2}^{2} a_{1} a_{3} a_{4}-x_{1}^{2} y_{2}^{2} a_{1} a_{4}^{2}-x_{1}^{2} y_{2}^{2} a_{1} a_{4} a_{5}-y_{1} y_{2}^{2} a_{1} a_{3} a_{4} a_{2}-$ $y_{1} y_{2}^{2} a_{1} a_{4}^{2} a_{2}-y_{1} y_{2}^{2} a_{1} a_{4} a_{5} a_{2}-x_{1}^{2} y_{2}^{2} a_{3} a_{4}-x_{1}^{2} y_{2}^{2} a_{3} a_{5}-y_{1} y_{2}^{2} a_{3}^{2} a_{2}-y_{1} y_{2}^{2} a_{3} a_{4} a_{2}+y_{1} y_{2}^{2} a_{3} a_{5} a_{2}-$ $y_{1} y_{2}^{2} a_{5}^{2} a_{2}-x_{1} y_{1}^{2} a_{1} a_{2}^{2}+x_{1} y_{1}^{2} a_{1} a_{2} a_{6}+x_{1} y_{1}^{2} a_{2} a_{6}+y_{1} y_{2}^{2} a_{2} a_{7}-y_{1} y_{2}^{2} a_{6} a_{7}-x_{1} x_{2} y_{2} a_{3}+x_{1} x_{2} y_{2} a_{5}+x_{2}^{2}$ $J_{13}=-y_{2}^{3} a_{1}^{2} a_{4}^{3}-y_{2}^{3} a_{1} a_{3} a_{4}^{2}-y_{2}^{3} a_{1} a_{4}^{3}-y_{2}^{3} a_{1} a_{4}^{2} a_{5}+x_{1}^{3} y_{2} a_{1} a_{4}-y_{2}^{3} a_{3}^{2} a_{4}-y_{2}^{3} a_{3} a_{4}^{2}+y_{2}^{3} a_{3} a_{4} a_{5}-y_{2}^{3} a_{4} a_{5}^{2}-$ $x_{1} y_{1} y_{2} a_{1} a_{4} a_{2}+x_{1} y_{1} y_{2} a_{1} a_{4} a_{6}+x_{1}^{3} y_{2} a_{3}+x_{1} y_{1} y_{2} a_{3} a_{6}+x_{1} y_{1} y_{2} a_{4} a_{6}+y_{2}^{3} a_{4} a_{7}-x_{1}^{2} x_{2}-x_{2} y_{1} a_{6}$ $J_{21}=-3 y_{1} y_{2}^{2} a_{1}^{2} a_{4}^{2} a_{2}-2 y_{1} y_{2}^{2} a_{1} a_{3} a_{4} a_{2}-3 y_{1} y_{2}^{2} a_{1} a_{4}^{2} a_{2}-2 y_{1} y_{2}^{2} a_{1} a_{4} a_{5} a_{2}+y_{1} y_{2}^{2} a_{1} a_{4}^{2} a_{6}+x_{1}^{5} a_{1}+$ $x_{1}^{2} y_{2}^{2} a_{3} a_{5}-x_{1}^{2} y_{2}^{2} a_{5}^{2}-y_{1} y_{2}^{2} a_{3}^{2} a_{2}-2 y_{1} y_{2}^{2} a_{3} a_{4} a_{2}+y_{1} y_{2}^{2} a_{3} a_{5} a_{2}-y_{1} y_{2}^{2} a_{5}^{2} a_{2}-x_{1} y_{1}^{2} a_{1} a_{2}^{2}+x_{1}^{3} y_{1} a_{1} a_{6}+$ $y_{1} y_{2}^{2} a_{4}^{2} a_{6}+y_{1} y_{2}^{2} a_{4} a_{5} a_{6}+x_{1} y_{1}^{2} a_{1} a_{2} a_{6}+x_{1}^{5}+x_{1}^{3} y_{1} a_{6}+x_{1} y_{1}^{2} a_{2} a_{6}+x_{1}^{2} y_{2}^{2} a_{7}+y_{1} y_{2}^{2} a_{2} a_{7}+x_{1} x_{2} y_{2} a_{3}+x_{2}^{2}$ $J_{22}=x_{1}^{3} y_{2} a_{1} a_{4}+x_{1}^{3} y_{2} a_{4}+x_{1}^{3} y_{2} a_{5}+x_{1} y_{1} y_{2} a_{5} a_{2}+y_{2}^{3} a_{4} a_{7}+x_{1}^{2} x_{2}+x_{2} y_{1} a_{2}$

$J_{23}=x_{1} y_{2}^{2} a_{3} a_{4}-x_{1} y_{2}^{2} a_{4} a_{5}-x_{1}^{4}-x_{1}^{2} y_{1} a_{2}-x_{1}^{2} y_{1} a_{6}-y_{1}^{2} a_{2} a_{6}+x_{2} y_{2} a_{4}$

$J_{31}=-x_{1}^{3} y_{2}^{2} a_{1}^{3} a_{4}^{2}-2 x_{1}^{3} y_{2}^{2} a_{1}^{2} a_{4}^{2}-2 x_{1}^{3} y_{2}^{2} a_{1}^{2} a_{4} a_{5}-2 x_{1} y_{1} y_{2}^{2} a_{1}^{2} a_{3} a_{4} a_{2}+3 x_{1} y_{1} y_{2}^{2} a_{1}^{2} a_{4}^{2} a_{2}+2 x_{1} y_{1} y_{2}^{2} a_{1}^{2} a_{4} a_{5} a_{2}-$ $y_{2}^{4} a_{1}^{2} a_{4}^{2} a_{7}+x_{1}^{6} a_{1}^{2}-x_{1}^{3} y_{2}^{2} a_{1} a_{3} a_{4}-x_{1}^{3} y_{2}^{2} a_{1} a_{4}^{2}+x_{1}^{3} y_{2}^{2} a_{1} a_{3} a_{5}-2 x_{1}^{3} y_{2}^{2} a_{1} a_{4} a_{5}-2 x_{1}^{3} y_{2}^{2} a_{1} a_{5}^{2}-x_{1}^{4} y_{1} a_{1}^{2} a_{2}-$ $x_{1} y_{1} y_{2}^{2} a_{1} a_{3}^{2} a_{2}+3 x_{1} y_{1} y_{2}^{2} a_{1} a_{4}^{2} a_{2}+4 x_{1} y_{1} y_{2}^{2} a_{1} a_{4} a_{5} a_{2}+x_{1} y_{1} y_{2}^{2} a_{1} a_{5}^{2} a_{2}+x_{1}^{4} y_{1} a_{1}^{2} a_{6}+x_{1} y_{1} y_{2}^{2} a_{1} a_{3} a_{4} a_{6}-$ $x_{1} y_{1} y_{2}^{2} a_{1} a_{4}^{2} a_{6}-x_{1} y_{1} y_{2}^{2} a_{1} a_{4} a_{5} a_{6}-y_{2}^{4} a_{1} a_{3} a_{4} a_{7}-y_{2}^{4} a_{1} a_{4}^{2} a_{7}-y_{2}^{4} a_{1} a_{4} a_{5} a_{7}+x_{1}^{2} x_{2} y_{2} a_{1}^{2} a_{4}-x_{1}^{3} y_{2}^{2} a_{3} a_{4}-$ $x_{1}^{3} y_{2}^{2} a_{3} a_{5}-x_{1}^{4} y_{1} a_{1} a_{2}-2 x_{2} y_{1} y_{2} a_{1}^{2} a_{4} a_{2}+2 x_{1} y_{1} y_{2}^{2} a_{3} a_{4} a_{2}+x_{1} y_{1} y_{2}^{2} a_{5}^{2} a_{2}+x_{1}^{2} y_{1}^{2} a_{1} a_{2}^{2}+x_{1}^{4} y_{1} a_{1} a_{6}+$ $x_{1} y_{1} y_{2}^{2} a_{3} a_{4} a_{6}-x_{1} y_{1} y_{2}^{2} a_{4}^{2} a_{6}+x_{1} y_{1} y_{2}^{2} a_{3} a_{5} a_{6}-2 x_{1} y_{1} y_{2}^{2} a_{4} a_{5} a_{6}-x_{1} y_{1} y_{2}^{2} a_{5}^{2} a_{6}-x_{1}^{2} y_{1}^{2} a_{1} a_{2} a_{6}+$ $2 x_{1}^{3} y_{2}^{2} a_{1} a_{7}-y_{2}^{4} a_{3}^{2} a_{7}-y_{2}^{4} a_{3} a_{4} a_{7}+y_{2}^{4} a_{3} a_{5} a_{7}-y_{2}^{4} a_{5}^{2} a_{7}-x_{1} y_{1} y_{2}^{2} a_{1} a_{2} a_{7}+x_{1} y_{1} y_{2}^{2} a_{1} a_{6} a_{7}-x_{1}^{6}+$ $x_{1}^{2} x_{2} y_{2} a_{1} a_{3}+x_{1}^{2} x_{2} y_{2} a_{1} a_{4}+x_{1}^{2} x_{2} y_{2} a_{1} a_{5}-x_{2} y_{1} y_{2} a_{1} a_{3} a_{2}-2 x_{2} y_{1} y_{2} a_{1} a_{4} a_{2}-x_{2} y_{1} y_{2} a_{1} a_{5} a_{2}+$ $x_{2} y_{1} y_{2} a_{1} a_{4} a_{6}-x_{1}^{2} y_{1}^{2} a_{2} a_{6}-x_{1} y_{1} y_{2}^{2} a_{2} a_{7}+x_{1} y_{1} y_{2}^{2} a_{6} a_{7}+y_{2}^{4} a_{7}^{2}-x_{2} y_{1} y_{2} a_{3} a_{2}+x_{2} y_{1} y_{2} a_{4} a_{6}+$ $x_{2} y_{1} y_{2} a_{5} a_{6}-x_{1} x_{2}^{2}$

$J_{32}=x_{1}^{4} y_{2} a_{1}^{2} a_{4}-x_{1}^{2} y_{1} y_{2} a_{1}^{2} a_{4} a_{2}-2 y_{1}^{2} y_{2} a_{1}^{2} a_{4} a_{2}^{2}+x_{1}^{4} y_{2} a_{1} a_{5}-x_{1}^{2} y_{1} y_{2} a_{1} a_{4} a_{2}-y_{1}^{2} y_{2} a_{1} a_{3} a_{2}^{2}-$ $2 y_{1}^{2} y_{2} a_{1} a_{4} a_{2}^{2}-y_{1}^{2} y_{2} a_{1} a_{5} a_{2}^{2}+x_{1}^{2} y_{1} y_{2} a_{1} a_{4} a_{6}+y_{1}^{2} y_{2} a_{1} a_{4} a_{2} a_{6}-x_{1}^{4} y_{2} a_{4}-x_{1}^{4} y_{2} a_{5}-x_{1}^{2} y_{1} y_{2} a_{5} a_{2}-$ $y_{1}^{2} y_{2} a_{3} a_{2}^{2}+x_{1}^{2} y_{1} y_{2} a_{4} a_{6}+x_{1}^{2} y_{1} y_{2} a_{5} a_{6}+y_{1}^{2} y_{2} a_{4} a_{2} a_{6}+y_{1}^{2} y_{2} a_{5} a_{2} a_{6}-x_{1} y_{2}^{3} a_{3} a_{7}-x_{1} y_{2}^{3} a_{4} a_{7}+x_{1}^{3} x_{2} a_{1}-$ $x_{1} x_{2} y_{1} a_{2}+x_{2} y_{2}^{2} a_{7}$

$J_{33}=x_{1}^{2} y_{2}^{2} a_{1}^{2} a_{4}^{2}-2 y_{1} y_{2}^{2} a_{1}^{2} a_{4}^{2} a_{2}+x_{1}^{2} y_{2}^{2} a_{1} a_{3} a_{4}+x_{1}^{2} y_{2}^{2} a_{1} a_{4}^{2}+x_{1}^{2} y_{2}^{2} a_{1} a_{4} a_{5}-y_{1} y_{2}^{2} a_{1} a_{3} a_{4} a_{2}-$ $2 y_{1} y_{2}^{2} a_{1} a_{4}^{2} a_{2}-y_{1} y_{2}^{2} a_{1} a_{4} a_{5} a_{2}+y_{1} y_{2}^{2} a_{1} a_{4}^{2} a_{6}-x_{1}^{5} a_{1}+x_{1}^{2} y_{2}^{2} a_{4} a_{5}+x_{1}^{2} y_{2}^{2} a_{5}^{2}-y_{1} y_{2}^{2} a_{3} a_{4} a_{2}-x_{1}^{3} y_{1} a_{1} a_{6}+$ $y_{1} y_{2}^{2} a_{4}^{2} a_{6}+y_{1} y_{2}^{2} a_{4} a_{5} a_{6}+x_{1}^{5}+x_{1}^{3} y_{1} a_{2}+x_{1} y_{1}^{2} a_{2} a_{6}-x_{1}^{2} y_{2}^{2} a_{7}-y_{1} y_{2}^{2} a_{6} a_{7}-x_{1} x_{2} y_{2} a_{4}-x_{1} x_{2} y_{2} a_{5}+x_{2}^{2}$

The seven coefficients $a_{1}, \ldots, a_{7}$ are subject to matrix factorisation conditions which take the form of twelve algebraic equations $f_{\alpha}(a)=0$ with

$f_{1}=-(1 / 3) a_{1} a_{3}^{2} a_{4} a_{6}-(1 / 3) a_{1} a_{3} a_{4}^{2} a_{6}+(1 / 3) a_{1} a_{3} a_{4} a_{5} a_{6}+(2 / 3) a_{1} a_{4}^{2} a_{5} a_{6}+(2 / 3) a_{1} a_{4} a_{5}^{2} a_{6}-$ $(2 / 3) a_{2} a_{3}^{3}-(1 / 3) a_{2} a_{3}^{2} a_{4}+2 a_{2} a_{3}^{2} a_{5}+(2 / 3) a_{2} a_{3} a_{4} a_{5}-2 a_{2} a_{3} a_{5}^{2}+(4 / 3) a_{2} a_{5}^{3}-(1 / 3) a_{3}^{2} a_{4} a_{6}-$ $(1 / 3) a_{3} a_{4}^{2} a_{6}+(2 / 3) a_{4}^{2} a_{5} a_{6}+(4 / 3) a_{4} a_{5}^{2} a_{6}+(2 / 3) a_{2} a_{3} a_{7}-(4 / 3) a_{2} a_{5} a_{7}-(5 / 3) a_{3} a_{6} a_{7}+(1 / 3) a_{5} a_{6} a_{7}$ $f_{2}=2 a_{1}^{2} a_{3} a_{4}^{2} a_{6}-4 a_{1}^{2} a_{4}^{2} a_{5} a_{6}+2 a_{1} a_{3} a_{4}^{2} a_{6}-4 a_{1} a_{4}^{2} a_{5} a_{6}-2 a_{2} a_{3}^{3}+6 a_{2} a_{3}^{2} a_{5}-6 a_{2} a_{3} a_{5}^{2}+4 a_{2} a_{5}^{3}-$ $2 a_{3} a_{4} a_{5} a_{6}+4 a_{4} a_{5}^{2} a_{6}+2 a_{2} a_{3} a_{7}-4 a_{2} a_{5} a_{7}-6 a_{3} a_{6} a_{7}$

$f_{3}=-7 a_{1}^{3} a_{2} a_{3} a_{4}^{2} a_{6}+11 a_{1}^{3} a_{2} a_{4}^{2} a_{5} a_{6}-4 a_{1}^{2} a_{2} a_{3}^{2} a_{4} a_{6}-12 a_{1}^{2} a_{2} a_{3} a_{4}^{2} a_{6}-2 a_{1}^{2} a_{2} a_{3} a_{4} a_{5} a_{6}+15 a_{1}^{2} a_{2} a_{4}^{2} a_{5} a_{6}+$ $14 a_{1}^{2} a_{2} a_{4} a_{5}^{2} a_{6}-a_{1}^{2} a_{4}^{3} a_{6}^{2}-a_{1} a_{2} a_{3}^{3} a_{6}-9 a_{1} a_{2} a_{3}^{2} a_{4} a_{6}-8 a_{1} a_{2} a_{3} a_{4}^{2} a_{6}+4 a_{1} a_{2} a_{3} a_{4} a_{5} a_{6}+4 a_{1} a_{2} a_{4}^{2} a_{5} a_{6}+$ $7 a_{1} a_{2} a_{4} a_{5}^{2} a_{6}+5 a_{1} a_{2} a_{5}^{3} a_{6}-2 a_{1} a_{4}^{3} a_{6}^{2}-2 a_{1} a_{4}^{2} a_{5} a_{6}^{2}-12 a_{1}^{2} a_{2} a_{4} a_{6} a_{7}-a_{2}^{2} a_{3}^{3}-2 a_{2}^{2} a_{3}^{2} a_{4}+a_{2}^{2} a_{3}^{2} a_{5}-$ $a_{2}^{2} a_{3} a_{5}^{2}-a_{2} a_{3}^{3} a_{6}-5 a_{2} a_{3}^{2} a_{4} a_{6}-3 a_{2} a_{3} a_{4}^{2} a_{6}+a_{2} a_{3}^{2} a_{5} a_{6}+a_{2} a_{3} a_{4} a_{5} a_{6}-a_{2} a_{3} a_{5}^{2} a_{6}+a_{2} a_{5}^{3} a_{6}-a_{4}^{3} a_{6}^{2}-$ $2 a_{4}^{2} a_{5} a_{6}^{2}-a_{4} a_{5}^{2} a_{6}^{2}-6 a_{1} a_{2} a_{3} a_{6} a_{7}-7 a_{1} a_{2} a_{4} a_{6} a_{7}-6 a_{1} a_{2} a_{5} a_{6} a_{7}+a_{1} a_{4} a_{6}^{2} a_{7}+a_{2}^{2} a_{3} a_{7}-a_{2} a_{3} a_{6} a_{7}-$ $a_{2} a_{5} a_{6} a_{7}+a_{4} a_{6}^{2} a_{7}+a_{5} a_{6}^{2} a_{7}$

$f_{4}=-(5 / 2) a_{1}^{3} a_{3} a_{4}^{2}+2 a_{1}^{3} a_{4}^{2} a_{5}-2 a_{1}^{2} a_{3}^{2} a_{4}-(9 / 2) a_{1}^{2} a_{3} a_{4}^{2}+2 a_{1}^{2} a_{3} a_{4} a_{5}+3 a_{1}^{2} a_{4}^{2} a_{5}-2 a_{1}^{2} a_{4} a_{5}^{2}-$ $(3 / 2) a_{1} a_{3}^{3}-4 a_{1} a_{3}^{2} a_{4}-2 a_{1} a_{3} a_{4}^{2}+(9 / 2) a_{1} a_{3}^{2} a_{5}+(11 / 2) a_{1} a_{3} a_{4} a_{5}+a_{1} a_{4}^{2} a_{5}-(9 / 2) a_{1} a_{3} a_{5}^{2}-4 a_{1} a_{4} a_{5}^{2}+$ 
$6 a_{1}^{2} a_{4} a_{7}-(3 / 2) a_{3}^{3}-2 a_{3}^{2} a_{4}+(9 / 2) a_{3}^{2} a_{5}+3 a_{3} a_{4} a_{5}-(9 / 2) a_{3} a_{5}^{2}-a_{4} a_{5}^{2}+a_{5}^{3}+3 a_{1} a_{3} a_{7}+6 a_{1} a_{4} a_{7}+$ $3 a_{1} a_{5} a_{7}+(7 / 2) a_{3} a_{7}+a_{4} a_{7}-a_{5} a_{7}$

$f_{5}=3 a_{1} a_{3}^{3} a_{4} a_{6}+a_{1} a_{3}^{2} a_{4}^{2} a_{6}-9 a_{1} a_{3}^{2} a_{4} a_{5} a_{6}+8 a_{1} a_{3} a_{4} a_{5}^{2} a_{6}-2 a_{1} a_{4}^{2} a_{5}^{2} a_{6}-4 a_{1} a_{4} a_{5}^{3} a_{6}+5 a_{2} a_{3}^{4}+$ $a_{2} a_{3}^{3} a_{4}-19 a_{2} a_{3}^{3} a_{5}-2 a_{2} a_{3}^{2} a_{4} a_{5}+25 a_{2} a_{3}^{2} a_{5}^{2}+a_{2} a_{3} a_{4} a_{5}^{2}-15 a_{2} a_{3} a_{5}^{3}-a_{2} a_{4} a_{5}^{3}+2 a_{2} a_{5}^{4}+3 a_{3}^{3} a_{4} a_{6}+$ $a_{3}^{2} a_{4}^{2} a_{6}-8 a_{3}^{2} a_{4} a_{5} a_{6}+7 a_{3} a_{4} a_{5}^{2} a_{6}-2 a_{4}^{2} a_{5}^{2} a_{6}-5 a_{4} a_{5}^{3} a_{6}-9 a_{1} a_{3} a_{4} a_{6} a_{7}+4 a_{1} a_{4}^{2} a_{6} a_{7}+9 a_{1} a_{4} a_{5} a_{6} a_{7}-$ $8 a_{2} a_{3}^{2} a_{7}+2 a_{2} a_{3} a_{4} a_{7}+11 a_{2} a_{3} a_{5} a_{7}+a_{2} a_{4} a_{5} a_{7}+11 a_{3}^{2} a_{6} a_{7}-11 a_{3} a_{4} a_{6} a_{7}+3 a_{4}^{2} a_{6} a_{7}-11 a_{3} a_{5} a_{6} a_{7}+$ $11 a_{4} a_{5} a_{6} a_{7}+3 a_{5}^{2} a_{6} a_{7}-2 a_{2} a_{7}^{2}+2 a_{6} a_{7}^{2}$

$f_{6}=3 a_{1}^{2} a_{4}^{2} a_{6}+2 a_{1} a_{3} a_{4} a_{6}+5 a_{1} a_{4}^{2} a_{6}+2 a_{1} a_{4} a_{5} a_{6}+a_{2} a_{3}^{2}+2 a_{2} a_{3} a_{4}-a_{2} a_{3} a_{5}+a_{2} a_{5}^{2}+2 a_{3} a_{4} a_{6}+$ $2 a_{4}^{2} a_{6}+a_{4} a_{5} a_{6}-a_{2} a_{7}+a_{6} a_{7}$

$f_{7}=a_{1} a_{2}-a_{1} a_{6}-a_{6}$

$f_{8}=-2 a_{1}^{2} a_{2} a_{4} a_{6}^{2}-a_{1} a_{2} a_{3} a_{6}^{2}-3 a_{1} a_{2} a_{4} a_{6}^{2}-a_{1} a_{2} a_{5} a_{6}^{2}-a_{2}^{2} a_{3} a_{6}-a_{2} a_{3} a_{6}^{2}-a_{2} a_{4} a_{6}^{2}+1$

$f_{9}=a_{1}^{2} a_{4}^{3} a_{7}+a_{1} a_{3} a_{4}^{2} a_{7}+a_{1} a_{4}^{3} a_{7}+a_{1} a_{4}^{2} a_{5} a_{7}+a_{3}^{2} a_{4} a_{7}+a_{3} a_{4}^{2} a_{7}-a_{3} a_{4} a_{5} a_{7}+a_{4} a_{5}^{2} a_{7}-a_{4} a_{7}^{2}+1$

$f_{10}=5 a_{1}^{2} a_{3} a_{4}^{2} a_{6}-a_{1}^{2} a_{4}^{2} a_{5} a_{6}+3 a_{1} a_{3}^{2} a_{4} a_{6}+8 a_{1} a_{3} a_{4}^{2} a_{6}+3 a_{1} a_{3} a_{4} a_{5} a_{6}-a_{1} a_{4}^{2} a_{5} a_{6}+a_{2} a_{3}^{3}+3 a_{2} a_{3}^{2} a_{4}+$ $a_{2} a_{5}^{3}+3 a_{3}^{2} a_{4} a_{6}+3 a_{3} a_{4}^{2} a_{6}+a_{3} a_{4} a_{5} a_{6}+a_{4} a_{5}^{2} a_{6}-a_{2} a_{3} a_{7}-a_{2} a_{5} a_{7}$

$f_{11}=-3 a_{1}^{2} a_{4} a_{6}^{2}-a_{1} a_{3} a_{6}^{2}-6 a_{1} a_{4} a_{6}^{2}-a_{1} a_{5} a_{6}^{2}-a_{2}^{2} a_{3}-a_{2} a_{3} a_{6}-a_{2} a_{4} a_{6}-a_{3} a_{6}^{2}-3 a_{4} a_{6}^{2}$

$f_{12}=a_{1}^{3} a_{4}^{3}+2 a_{1}^{2} a_{4}^{3}+3 a_{1}^{2} a_{4}^{2} a_{5}+a_{1} a_{3} a_{4}^{2}+a_{1} a_{4}^{3}+3 a_{1} a_{4}^{2} a_{5}+3 a_{1} a_{4} a_{5}^{2}+a_{3} a_{4}^{2}+a_{3} a_{4} a_{5}+a_{4} a_{5}^{2}+a_{5}^{3}-$

$2 a_{1} a_{4} a_{7}-a_{3} a_{7}-a_{4} a_{7}-a_{5} a_{7}$

These twelve equations are solvable, and the quantum dimensions, subject to the matrix factorisation conditions, are given by

$q_{L}(Q)=a_{1} a_{4} a_{6}+a_{2} a_{3}+a_{4} a_{6}+a_{5} a_{6}$

$q_{R}(Q)=\left(462 a_{1} a_{5} a_{6}^{2} a_{7}^{2}+603 a_{1}^{3} a_{6}^{2}-2002 a_{2}^{2} a_{3} a_{7}^{2}+158 a_{2}^{2} a_{4} a_{7}^{2}-853 a_{2}^{2} a_{5} a_{7}^{2}-898 a_{2} a_{3} a_{6} a_{7}^{2}-\right.$ $2784 a_{2} a_{4} a_{6} a_{7}^{2}-136 a_{2} a_{5} a_{6} a_{7}^{2}+214 a_{3} a_{6}^{2} a_{7}^{2}-1294 a_{4} a_{6}^{2} a_{7}^{2}+1111 a_{5} a_{6}^{2} a_{7}^{2}+2646 a_{1}^{2} a_{6}^{2}-261 a_{1} a_{6}^{2}-$ $\left.291 a_{2}^{2}-301 a_{2} a_{6}-2095 a_{6}^{2}\right) / 764$

Note that these expressions result after reduction by the ideal spanned by the $f_{\alpha}$, hence the quantum dimensions of this defect are non-zero numbers after inserting any special solution to the equations $f_{\alpha}(a)=0$.

(3) A rank 6 orbifold equivalence between $Z_{13}$ and $Q_{11}$, which could be worse:

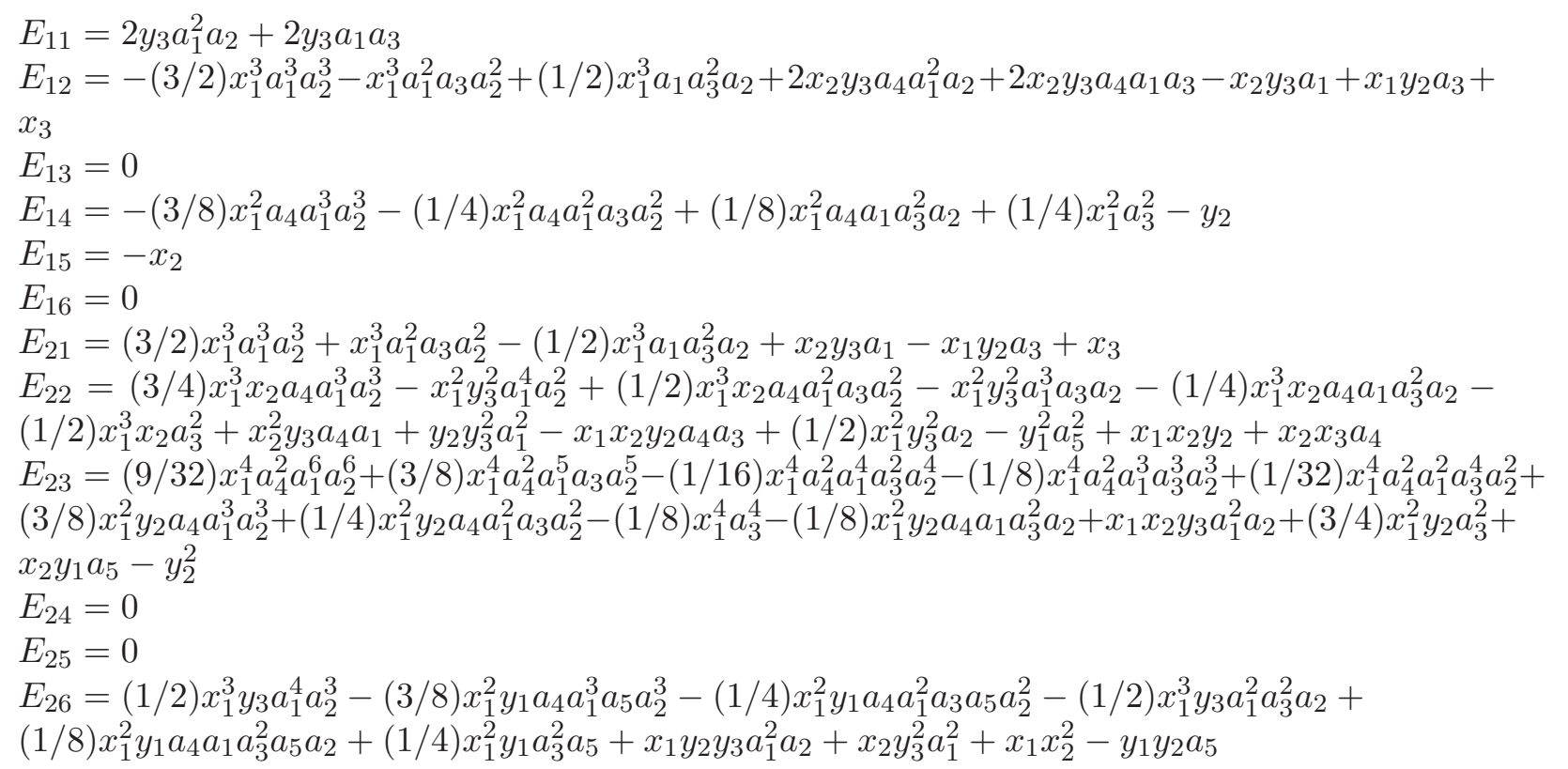


$E_{31}=0$

$E_{32}=(9 / 32) x_{1}^{4} a_{4}^{2} a_{1}^{6} a_{2}^{6}+(3 / 8) x_{1}^{4} a_{4}^{2} a_{1}^{5} a_{3} a_{2}^{5}-(1 / 16) x_{1}^{4} a_{4}^{2} a_{1}^{4} a_{3}^{2} a_{2}^{4}-(1 / 8) x_{1}^{4} a_{4}^{2} a_{1}^{3} a_{3}^{3} a_{2}^{3}+(1 / 32) x_{1}^{4} a_{4}^{2} a_{1}^{2} a_{3}^{4} a_{2}^{2}+$ $(3 / 8) x_{1}^{2} y_{2} a_{4} a_{1}^{3} a_{2}^{3}+(1 / 4) x_{1}^{2} y_{2} a_{4} a_{1}^{2} a_{3} a_{2}^{2}-(1 / 8) x_{1}^{4} a_{3}^{4}-(1 / 8) x_{1}^{2} y_{2} a_{4} a_{1} a_{3}^{2} a_{2}+x_{1} x_{2} y_{3} a_{1}^{2} a_{2}+(3 / 4) x_{1}^{2} y_{2} a_{3}^{2}-$ $x_{2} y_{1} a_{5}-y_{2}^{2}$

$E_{33}=-(3 / 4) x_{1}^{2} y_{3} a_{4} a_{1}^{5} a_{2}^{4}-(1 / 2) x_{1}^{2} y_{3} a_{4} a_{1}^{4} a_{3} a_{2}^{3}+(1 / 4) x_{1}^{2} y_{3} a_{4} a_{1}^{3} a_{3}^{2} a_{2}^{2}+3 x_{1}^{2} y_{3} a_{1}^{4} a_{2}^{3}+2 x_{1}^{2} y_{3} a_{1}^{3} a_{3} a_{2}^{2}-$ $(1 / 2) x_{1}^{2} y_{3} a_{1}^{2} a_{3}^{2} a_{2}-2 y_{2} y_{3} a_{1}^{2} a_{2}-2 y_{2} y_{3} a_{1} a_{3}+x_{2}^{2}$

$E_{34}=(3 / 2) x_{1}^{3} a_{1}^{3} a_{2}^{3}+x_{1}^{3} a_{1}^{2} a_{3} a_{2}^{2}-(1 / 2) x_{1}^{3} a_{1} a_{3}^{2} a_{2}+x_{2} y_{3} a_{1}-x_{1} y_{2} a_{3}+x_{3}$

$E_{35}=0$

$E_{36}=-(3 / 8) x_{1}^{2} x_{2} a_{4} a_{1}^{3} a_{2}^{3}+2 x_{1} y_{3}^{2} a_{1}^{4} a_{2}^{2}-(1 / 4) x_{1}^{2} x_{2} a_{4} a_{1}^{2} a_{3} a_{2}^{2}+2 x_{1} y_{3}^{2} a_{1}^{3} a_{3} a_{2}+(1 / 8) x_{1}^{2} x_{2} a_{4} a_{1} a_{3}^{2} a_{2}-$ $2 y_{1} y_{3} a_{1}^{2} a_{5} a_{2}+(1 / 4) x_{1}^{2} x_{2} a_{3}^{2}-2 y_{1} y_{3} a_{1} a_{3} a_{5}-x_{2} y_{2}$

$E_{41}=-(3 / 8) x_{1}^{2} a_{4} a_{1}^{3} a_{2}^{3}-(1 / 4) x_{1}^{2} a_{4} a_{1}^{2} a_{3} a_{2}^{2}+(1 / 8) x_{1}^{2} a_{4} a_{1} a_{3}^{2} a_{2}+(1 / 4) x_{1}^{2} a_{3}^{2}-y_{2}$

$E_{42}=-(3 / 8) x_{1}^{2} x_{2} a_{4}^{2} a_{1}^{3} a_{2}^{3}-(1 / 4) x_{1}^{2} x_{2} a_{4}^{2} a_{1}^{2} a_{3} a_{2}^{2}+(1 / 8) x_{1}^{2} x_{2} a_{4}^{2} a_{1} a_{3}^{2} a_{2}+(1 / 4) x_{1}^{2} x_{2} a_{4} a_{3}^{2}-x_{2} y_{2} a_{4}$

$E_{43}=-(3 / 2) x_{1}^{3} a_{1}^{3} a_{2}^{3}-x_{1}^{3} a_{1}^{2} a_{3} a_{2}^{2}+(1 / 2) x_{1}^{3} a_{1} a_{3}^{2} a_{2}-x_{2} y_{3} a_{1}+x_{1} y_{2} a_{3}+x_{3}$

$E_{44}=-y_{3}^{2} a_{1}^{2}-x_{1} x_{2}$

$E_{45}=x_{1} y_{3} a_{1}^{2} a_{2}-y_{1} a_{5}$

$E_{46}=0$

$E_{51}=-x_{2}$

$E_{52}=-x_{2}^{2} a_{4}$

$E_{53}=0$

$E_{54}=x_{1} y_{3} a_{1}^{2} a_{2}+y_{1} a_{5}$

$E_{55}=-x_{1}^{2} a_{1}^{2} a_{2}^{2}+y_{2}$

$E_{56}=-(3 / 2) x_{1}^{3} a_{1}^{3} a_{2}^{3}-x_{1}^{3} a_{1}^{2} a_{3} a_{2}^{2}+(1 / 2) x_{1}^{3} a_{1} a_{3}^{2} a_{2}-x_{2} y_{3} a_{1}+x_{1} y_{2} a_{3}+x_{3}$

$E_{61}=-x_{1} x_{2} a_{3}$

$E_{62}=(1 / 2) x_{1}^{3} y_{3} a_{1}^{4} a_{2}^{3}+(3 / 8) x_{1}^{2} y_{1} a_{4} a_{1}^{3} a_{5} a_{2}^{3}+(1 / 4) x_{1}^{2} y_{1} a_{4} a_{1}^{2} a_{3} a_{5} a_{2}^{2}-(1 / 2) x_{1}^{3} y_{3} a_{1}^{2} a_{3}^{2} a_{2}-$

$(1 / 8) x_{1}^{2} y_{1} a_{4} a_{1} a_{3}^{2} a_{5} a_{2}-(1 / 4) x_{1}^{2} y_{1} a_{3}^{2} a_{5}+x_{1} y_{2} y_{3} a_{1}^{2} a_{2}+x_{2} y_{3}^{2} a_{1}^{2}-x_{1} x_{2}^{2} a_{4} a_{3}+x_{1} x_{2}^{2}+y_{1} y_{2} a_{5}$

$E_{63}=-(3 / 8) x_{1}^{2} x_{2} a_{4} a_{1}^{3} a_{2}^{3}-(1 / 4) x_{1}^{2} x_{2} a_{4} a_{1}^{2} a_{3} a_{2}^{2}+(1 / 8) x_{1}^{2} x_{2} a_{4} a_{1} a_{3}^{2} a_{2}+2 y_{1} y_{3} a_{1}^{2} a_{5} a_{2}+(1 / 4) x_{1}^{2} x_{2} a_{3}^{2}+$

$2 y_{1} y_{3} a_{1} a_{3} a_{5}+x_{1} y_{3}^{2} a_{2}-x_{2} y_{2}$

$E_{64}=x_{1}^{2} y_{3} a_{1}^{2} a_{3} a_{2}+x_{1} y_{1} a_{3} a_{5}$

$E_{65}=(3 / 2) x_{1}^{3} a_{1}^{3} a_{2}^{3}-(1 / 2) x_{1}^{3} a_{1} a_{3}^{2} a_{2}+x_{2} y_{3} a_{1}+x_{3}$

$E_{66}=x_{1}^{4} a_{1}^{4} a_{2}^{4}+(3 / 2) x_{1}^{4} a_{1}^{3} a_{3} a_{2}^{3}-(1 / 2) x_{1}^{4} a_{1} a_{3}^{3} a_{2}+2 y_{3}^{3} a_{1}^{4} a_{2}+2 y_{3}^{3} a_{1}^{3} a_{3}+x_{1}^{2} y_{2} a_{1}^{2} a_{2}^{2}+2 x_{1} x_{2} y_{3} a_{1}^{2} a_{2}+$ $x_{1} x_{2} y_{3} a_{1} a_{3}+x_{1} x_{3} a_{3}+y_{2}^{2}$

$J_{11}=-(3 / 4) x_{1}^{3} x_{2} a_{4} a_{1}^{3} a_{2}^{3}+x_{1}^{2} y_{3}^{2} a_{1}^{4} a_{2}^{2}-(1 / 2) x_{1}^{3} x_{2} a_{4} a_{1}^{2} a_{3} a_{2}^{2}+x_{1}^{2} y_{3}^{2} a_{1}^{3} a_{3} a_{2}+(1 / 4) x_{1}^{3} x_{2} a_{4} a_{1} a_{3}^{2} a_{2}+$ $(1 / 2) x_{1}^{3} x_{2} a_{3}^{2}-x_{2}^{2} y_{3} a_{4} a_{1}-y_{2} y_{3}^{2} a_{1}^{2}+x_{1} x_{2} y_{2} a_{4} a_{3}-(1 / 2) x_{1}^{2} y_{3}^{2} a_{2}+y_{1}^{2} a_{5}^{2}-x_{1} x_{2} y_{2}-x_{2} x_{3} a_{4}$

$J_{12}=-(3 / 2) x_{1}^{3} a_{1}^{3} a_{2}^{3}-x_{1}^{3} a_{1}^{2} a_{3} a_{2}^{2}+(1 / 2) x_{1}^{3} a_{1} a_{3}^{2} a_{2}+2 x_{2} y_{3} a_{4} a_{1}^{2} a_{2}+2 x_{2} y_{3} a_{4} a_{1} a_{3}-x_{2} y_{3} a_{1}+x_{1} y_{2} a_{3}+$ $x_{3}$

$J_{13}=-(3 / 8) x_{1}^{2} x_{2} a_{4}^{2} a_{1}^{3} a_{2}^{3}-(1 / 4) x_{1}^{2} x_{2} a_{4}^{2} a_{1}^{2} a_{3} a_{2}^{2}+(1 / 8) x_{1}^{2} x_{2} a_{4}^{2} a_{1} a_{3}^{2} a_{2}+(1 / 4) x_{1}^{2} x_{2} a_{4} a_{3}^{2}-x_{2} y_{2} a_{4}$ $J_{14}=-(9 / 32) x_{1}^{4} a_{4}^{2} a_{1}^{6} a_{2}^{6}-(3 / 8) x_{1}^{4} a_{4}^{2} a_{1}^{5} a_{3} a_{2}^{5}+(1 / 16) x_{1}^{4} a_{4}^{2} a_{1}^{4} a_{3}^{2} a_{2}^{4}+(1 / 8) x_{1}^{4} a_{4}^{2} a_{1}^{3} a_{3}^{3} a_{2}^{3}-(1 / 32) x_{1}^{4} a_{4}^{2} a_{1}^{2} a_{3}^{4} a_{2}^{2}-$ $(3 / 8) x_{1}^{2} y_{2} a_{4} a_{1}^{3} a_{2}^{3}-(1 / 4) x_{1}^{2} y_{2} a_{4} a_{1}^{2} a_{3} a_{2}^{2}+(1 / 8) x_{1}^{4} a_{3}^{4}+(1 / 8) x_{1}^{2} y_{2} a_{4} a_{1} a_{3}^{2} a_{2}-x_{1} x_{2} y_{3} a_{1}^{2} a_{2}-(3 / 4) x_{1}^{2} y_{2} a_{3}^{2}-$ $x_{2} y_{1} a_{5}+y_{2}^{2}$

$J_{15}=-(1 / 2) x_{1}^{3} y_{3} a_{1}^{4} a_{2}^{3}+(3 / 8) x_{1}^{2} y_{1} a_{4} a_{1}^{3} a_{5} a_{2}^{3}+(1 / 4) x_{1}^{2} y_{1} a_{4} a_{1}^{2} a_{3} a_{5} a_{2}^{2}+(1 / 2) x_{1}^{3} y_{3} a_{1}^{2} a_{3}^{2} a_{2}-$ $(1 / 8) x_{1}^{2} y_{1} a_{4} a_{1} a_{3}^{2} a_{5} a_{2}-(1 / 4) x_{1}^{2} y_{1} a_{3}^{2} a_{5}-x_{1} y_{2} y_{3} a_{1}^{2} a_{2}-x_{2} y_{3}^{2} a_{1}^{2}+x_{1} x_{2}^{2} a_{4} a_{3}-x_{1} x_{2}^{2}+y_{1} y_{2} a_{5}$

$J_{16}=-x_{2}^{2} a_{4}$

$J_{21}=(3 / 2) x_{1}^{3} a_{1}^{3} a_{2}^{3}+x_{1}^{3} a_{1}^{2} a_{3} a_{2}^{2}-(1 / 2) x_{1}^{3} a_{1} a_{3}^{2} a_{2}+x_{2} y_{3} a_{1}-x_{1} y_{2} a_{3}+x_{3}$

$J_{22}=-2 y_{3} a_{1}^{2} a_{2}-2 y_{3} a_{1} a_{3}$

$J_{23}=(3 / 8) x_{1}^{2} a_{4} a_{1}^{3} a_{2}^{3}+(1 / 4) x_{1}^{2} a_{4} a_{1}^{2} a_{3} a_{2}^{2}-(1 / 8) x_{1}^{2} a_{4} a_{1} a_{3}^{2} a_{2}-(1 / 4) x_{1}^{2} a_{3}^{2}+y_{2}$

$J_{24}=0$ 


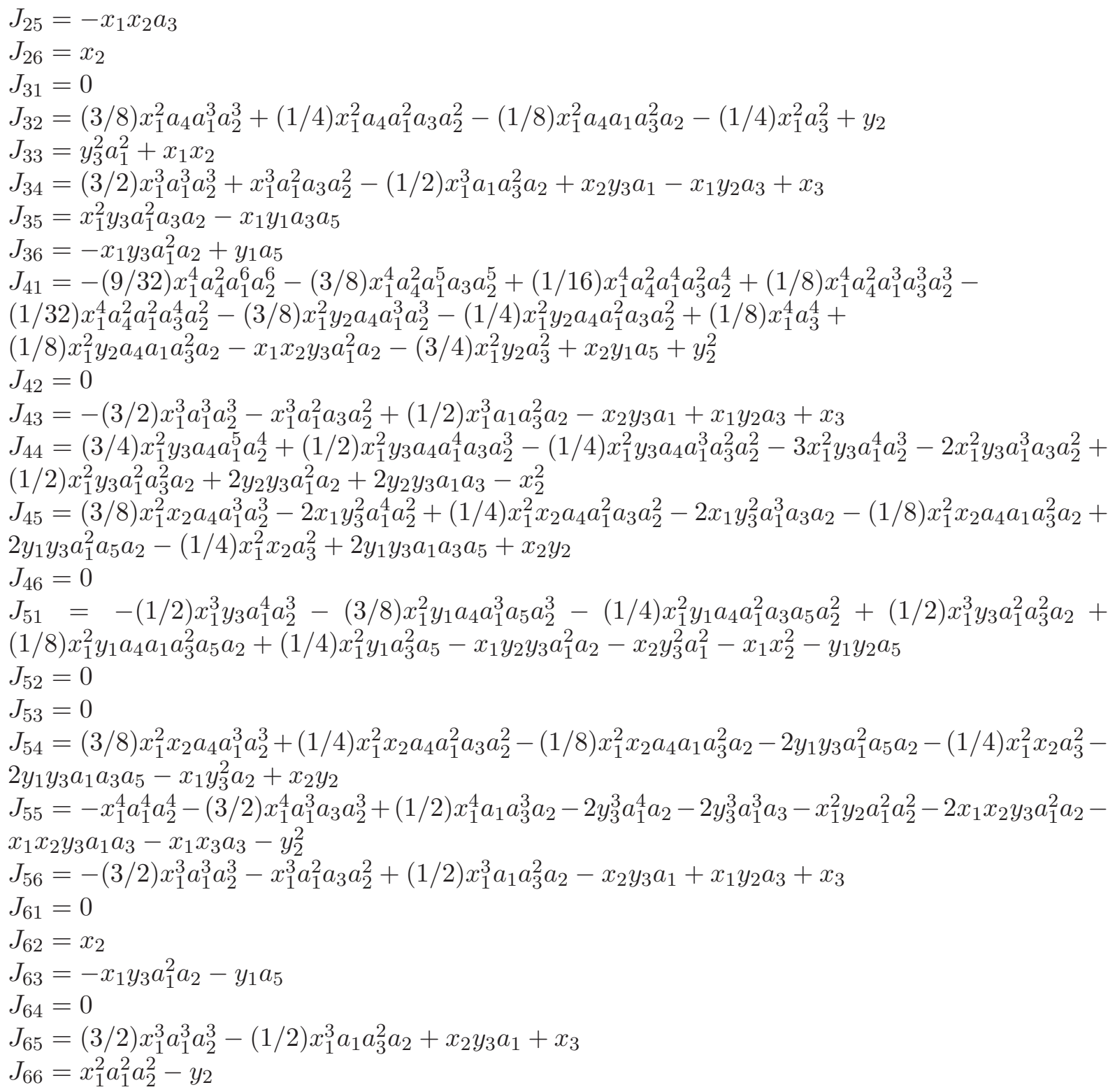

The five coefficients $a_{1}, \ldots, a_{5}$ are subject to thirty-seven relatively simple conditions $f_{\alpha}(a)=0$ with

$f_{1}=a_{1}^{2}+a_{5}^{2}$

$f_{2}=-3 a_{1} a_{2} a_{4}+52 a_{1} a_{3} a_{5}^{2}-7 a_{3} a_{4}-10$

$f_{3}=-1839 a_{1} a_{2} a_{3}+30 a_{2}^{2} a_{5}^{2}+835 a_{3}^{2}+72 a_{4}^{4}$

$f_{4}=94888 a_{1} a_{4} a_{5}^{2}-6675 a_{2}^{3}+7504 a_{3} a_{4}^{3}+41908 a_{4}^{2}$

$f_{5}=-159 a_{1} a_{2} a_{4}+52 a_{3}^{2} a_{4}^{2}+383 a_{3} a_{4}+445$

$f_{6}=83 a_{1} a_{2} a_{5}^{2}+14 a_{2} a_{3} a_{4}^{2}+53 a_{2} a_{4}-75 a_{3} a_{5}^{2}$

$f_{7}=225 a_{1} a_{2} a_{4}^{2}+2314 a_{1} a_{5}^{2}+187 a_{3} a_{4}^{2}+724 a_{4}$

$f_{8}=-36 a_{1} a_{2} a_{3}+15 a_{2}^{2} a_{5}^{2}+8 a_{3}^{3} a_{4}+47 a_{3}^{2}$

$f_{9}=2 a_{1} a_{3}^{2} a_{4}+78 a_{1} a_{3}-15 a_{2}^{2} a_{4}^{2}-81 a_{2} a_{5}^{2}$

$f_{10}=10 a_{1} a_{2} a_{3} a_{4}+13 a_{1} a_{2}+6 a_{3}^{2} a_{4}+29 a_{3}$

$f_{11}=33 a_{1} a_{2}^{2} a_{4}-27 a_{2} a_{3} a_{4}-72 a_{2}-52 a_{3}^{2} a_{5}^{2}$ 


$$
\begin{aligned}
& f_{12}=-145863 a_{2}^{3}+47444 a_{3}^{4}-31896 a_{3} a_{4}^{3}-82080 a_{4}^{2} \\
& f_{13}=1892 a_{1} a_{3}^{3}-648 a_{1} a_{4}^{3}+228 a_{2}^{2} a_{3} a_{4}-2223 a_{2}^{2} \\
& f_{14}=8 a_{1} a_{3} a_{4}^{2}+44 a_{1} a_{4}+a_{2}^{2} a_{3}^{2}-92 a_{5}^{4} \\
& f_{15}=1311 a_{1} a_{2} a_{3}^{2}+342 a_{2}^{3} a_{4}-211 a_{3}^{3}+180 a_{4}^{3} \\
& f_{16}=-4926 a_{1} a_{2} a_{4}^{2}+1157 a_{2}^{3} a_{3}+102 a_{3} a_{4}^{2}+7968 a_{4} \\
& f_{17}=41 a_{2}^{4}-132 a_{2} a_{4}^{2}-408 a_{3} a_{4} a_{5}^{2}-784 a_{5}^{2} \\
& f_{18}=1002 a_{1} a_{2}^{3}-12 a_{1} a_{4}^{2}-469 a_{2}^{2} a_{3}+1224 a_{4} a_{5}^{4} \\
& f_{19}=7 a_{1} a_{3} a_{4}+10 a_{1}-3 a_{2} a_{4} a_{5}^{2}+52 a_{3} a_{5}^{4} \\
& f_{20}=-2 a_{1} a_{3} a_{5}^{2}+2 a_{2} a_{5}^{4}-1 \\
& f_{21}=67716 a_{1} a_{5}^{4}-959 a_{2} a_{3}^{3}-1584 a_{2} a_{4}^{3}+23256 a_{4} a_{5}^{2} \\
& f_{22}=-71 a_{1} a_{2}^{2}+116 a_{2} a_{3}^{2} a_{4}+403 a_{2} a_{3}+48 a_{4}^{3} a_{5}^{2} \\
& f_{23}=3649 a_{2} a_{3}^{3}+9999 a_{2} a_{4}^{3}+33858 a_{3} a_{4}^{2} a_{5}^{2}+55575 a_{4} a_{5}^{2} \\
& f_{24}=654 a_{1} a_{3} a_{4}^{2}+17604 a_{1} a_{4}+1157 a_{2}^{2} a_{3}^{2}+10350 a_{2} a_{4}^{2} a_{5}^{2} \\
& f_{25}=283176 a_{1} a_{4}^{2} a_{5}^{2}-170487 a_{2}^{3} a_{4}-166964 a_{3}^{3}+46476 a_{4}^{3} \\
& f_{26}=123 a_{1} a_{2} a_{5}^{2}+15 a_{2} a_{3} a_{4}^{2}+106 a_{3}^{2} a_{4} a_{5}^{2}+514 a_{3} a_{5}^{2} \\
& f_{27}=-6 a_{1} a_{3}^{2} a_{4}-29 a_{1} a_{3}+10 a_{2} a_{3} a_{4} a_{5}^{2}+13 a_{2} a_{5}^{2} \\
& f_{28}=3 a_{1} a_{2}+3 a_{2}^{2} a_{4} a_{5}^{2}-a_{3}^{2} a_{4}-7 a_{3} \\
& f_{29}=246 a_{1} a_{2} a_{4} a_{5}^{2}-39 a_{2} a_{4}^{2}-128 a_{3} a_{4} a_{5}^{2}-623 a_{5}^{2} \\
& f_{30}=33 a_{1} a_{2}^{2}+36 a_{2} a_{3}^{2} a_{4}+129 a_{2} a_{3}+40 a_{3}^{3} a_{5}^{2} \\
& f_{31}=-22 a_{1} a_{3}^{3}-24 a_{2}^{2} a_{3} a_{4}-9 a_{2}^{2}+54 a_{2} a_{3}^{2} a_{5}^{2} \\
& f_{32}=2 a_{1} a_{2} a_{3}^{2}-6 a_{2}^{3} a_{4}+45 a_{2}^{2} a_{3} a_{5}^{2}+3 a_{3}^{3} \\
& f_{33}=-40 a_{1} a_{2}^{2} a_{3}+123 a_{2}^{3} a_{5}^{2}+51 a_{2} a_{3}^{2}+48 a_{4}^{2} a_{5}^{2} \\
& f_{34}=9 a_{1} a_{2}^{2} a_{5}^{2}+7 a_{1} a_{3}^{2}+6 a_{2}^{2} a_{4}-18 a_{2} a_{3} a_{5}^{2} \\
& f_{35}=-329 a_{1} a_{3} a_{4}+89 a_{1}+78 a_{2}^{2} a_{4}^{3}+453 a_{2} a_{4} a_{5}^{2} \\
& f_{36}=-3211 a_{1} a_{2} a_{3}+393 a_{2}^{3} a_{4}^{2}+2637 a_{2}^{2} a_{5}^{2}+306 a_{3}^{3} a_{4}+1765 a_{3}^{2} \\
& f_{37}=-5112 a_{1} a_{4} a_{5}^{2}+19 a_{2}^{3}+612 a_{4}^{2}+15008 a_{5}^{6}
\end{aligned}
$$

The quantum dimensions of $Q$ are:

$$
\begin{aligned}
& q_{L}(Q)=(24 / 13) a_{2} a_{4} a_{5}^{3}-(4 / 13) a_{1} a_{3} a_{4} a_{5}+(50 / 13) a_{1} a_{5} \\
& q_{R}(Q)=-2 a_{1} a_{2} a_{5}-2 a_{3} a_{5} .
\end{aligned}
$$

(4) A rank 4 orbifold equivalence between $S_{11}$ and $W_{13}$ :

$$
\begin{aligned}
& E_{11}=x_{1} y_{3}-y_{1} \\
& E_{12}=-x_{2} y_{3}^{2}+x_{1}^{2}+x_{2} x_{3} \\
& E_{13}=-x_{2}+y_{2} \\
& E_{14}=0 \\
& E_{21}=-y_{3}^{2}-x_{3} \\
& E_{22}=-x_{1} y_{3}-y_{1} \\
& E_{23}=0 \\
& E_{24}=-x_{2}+y_{2} \\
& E_{31}=x_{2}^{3}+x_{2}^{2} y_{2}+x_{2} y_{2}^{2}+y_{2}^{3}-x_{3} y_{3}^{2} \\
& E_{32}=-x_{1} y_{3}^{3}-y_{1} y_{3}^{2} \\
& E_{33}=-x_{1} y_{3}-y_{1} \\
& E_{34}=y_{2} y_{3}^{2}-x_{1}^{2}-x_{2} x_{3} \\
& E_{41}=0 \\
& E_{42}=y_{3}^{4}+x_{2}^{3}+x_{2}^{2} y_{2}+x_{2} y_{2}^{2}+y_{2}^{3}
\end{aligned}
$$




$$
\begin{aligned}
& E_{43}=y_{3}^{2}+x_{3} \\
& E_{44}=x_{1} y_{3}-y_{1} \\
& J_{11}=-x_{1} y_{3}-y_{1} \\
& J_{12}=y_{2} y_{3}^{2}-x_{1}^{2}-x_{2} x_{3} \\
& J_{13}=x_{2}-y_{2} \\
& J_{14}=0 \\
& J_{21}=y_{3}^{2}+x_{3} \\
& J_{22}=x_{1} y_{3}-y_{1} \\
& J_{23}=0 \\
& J_{24}=x_{2}-y_{2} \\
& J_{31}=-y_{3}^{4}-x_{2}^{3}-x_{2}^{2} y_{2}-x_{2} y_{2}^{2}-y_{2}^{3} \\
& J_{32}=-x_{1} y_{3}^{3}+y_{1} y_{3}^{2} \\
& J_{33}=x_{1} y_{3}-y_{1} \\
& J_{34}=-x_{2} y_{3}^{2}+x_{1}^{2}+x_{2} x_{3} \\
& J_{41}=0 \\
& J_{42}=-x_{2}^{3}-x_{2}^{2} y_{2}-x_{2} y_{2}^{2}-y_{2}^{3}+x_{3} y_{3}^{2} \\
& J_{43}=-y_{3}^{2}-x_{3} \\
& J_{44}=-x_{1} y_{3}-y_{1}
\end{aligned}
$$

This rather simple $Q$ does not depend on any coefficients, although more general orbifold equivalences between $S_{11}$ and $W_{13}$ can be found.

Its quantum dimensions are $q_{L}(Q)=-2$ and $q_{R}(Q)=-1$.

(5) A rank 3 orbifold equivalence between a chain and a loop at central charge $\hat{c}=\frac{6}{5}$ :

$$
\begin{aligned}
E_{11} & =2 a_{1} x_{1}^{4}+2 a_{1} x_{1} y_{2}^{2} \\
E_{12} & =a_{1} x_{1}^{3} y_{2}+a_{1} y_{2}^{3}+y_{1} \\
E_{13} & =a_{1} x_{1}^{5}+a_{1} x_{1}^{2} y_{2}^{2}+x_{2} \\
E_{21} & =-2 a_{1} x_{1}^{3} y_{2}-a_{1} y_{2}^{3}+y_{1} \\
E_{22} & =-a_{1} x_{1}^{2} y_{2}^{2}+x_{2} \\
E_{23} & =-a_{1} x_{1}^{4} y_{2}+x_{1} y_{1} \\
E_{31} & =-a_{1} x_{1}^{5}+x_{2} \\
E_{32} & =-x_{1} y_{1} \\
E_{33} & =-y_{1} y_{2} \\
J_{11} & =a_{1} x_{1}^{5} y_{1} y_{2}-a_{1} x_{1}^{2} y_{1} y_{2}^{3}-x_{1}^{2} y_{1}^{2}+x_{2} y_{1} y_{2} \\
J_{12} & =a_{1} x_{1}^{6} y_{1}-a_{1} y_{1} y_{2}^{4}+x_{1} x_{2} y_{1}-y_{1}^{2} y_{2} \\
J_{13} & =a_{1}^{2}+1 x_{1}^{10}+a_{1} x_{1}^{5} x_{2}-a_{1} x_{1} y_{1} y_{2}^{3}-x_{1} y_{1}^{2}+x_{2}^{2} \\
J_{21} & =-a_{1}^{2} x_{1}^{9} y_{2}+a_{1} x_{1}^{6} y_{1}+a_{1} x_{1}^{4} x_{2} y_{2}+2 a_{1} x_{1}^{3} y_{1} y_{2}^{2}+a_{1} y_{1} y_{2}^{4}-x_{1} x_{2} y_{1}-y_{1}^{2} y_{2} \\
J_{22} & =x_{1}^{10}-a_{1}^{2} x_{1}^{7} y_{2}^{2}+2 a_{1} x_{1}^{4} y_{1} y_{2}+a_{1} x_{1}^{2} x_{2} y_{2}^{2}+2 a_{1} x_{1} y_{1} y_{2}^{3}+x_{2}^{2} \\
J_{23} & =a_{1}^{2} x_{1}^{5} y_{2}^{3}+a_{1}^{2} x_{1}^{2} y_{2}^{5}+a_{1} x_{1}^{5} y_{1}+2 a_{1} x_{1}^{3} x_{2} y_{2}+a_{1} x_{1}^{2} y_{1} y_{2}^{2}+a_{1} x_{2} y_{2}^{3}-x_{2} y_{1} \\
J_{31} & =\left(a_{1}^{2}+1\right) x_{1}^{10}+a_{1}^{2} x_{1}^{7} y_{2}^{2}-a_{1} x_{1}^{5} x_{2}-2 a_{1} x_{1}^{4} y_{1} y_{2}-a_{1} x_{1}^{2} x_{2} y_{2}^{2}-a_{1} x_{1} y_{1} y_{2}^{3}+x_{1} y_{1}^{2}+x_{2}^{2} \\
J_{32} & =a_{1}^{2} x_{1}^{8} y_{2}+a_{1}^{2} x_{1}^{5} y_{2}^{3}-a_{1} x_{1}^{5} y_{1}-a_{1} x_{1}^{3} x_{2} y_{2}-2 a_{1} x_{1}^{2} y_{1} y_{2}^{2}-a_{1} x_{2} y_{2}^{3}-x_{2} y_{1} \\
J_{33} & =-a_{1}^{2} x_{1}^{3} y_{2}^{4}-a_{1}^{2} y_{2}^{6}-2 a_{1} x_{1}^{4} x_{2}-a_{1} x_{1}^{3} y_{1} y_{2}-2 a_{1} x_{1} x_{2} y_{2}^{2}+y_{1}^{2}
\end{aligned}
$$

This contains only a single coefficient $a_{1}$ which has to satisfy $a_{1}^{2}=-1$.

The quantum dimensions of this defect are $q_{L}(Q)=-2$ and $q_{R}(Q)=-3$. 


\section{References}

[1] V.I. Arnold, V.V. Goryunov, O.V. Lyashko, V.A.Vasilev, Singularity Theory I, Springer 1998

[2] P. Berglund, T. Hübsch, A generalized construction of mirror manifolds, Nucl. Phys. B393 (1993) 377, arXiv:hep-th/9201014

[3] I. Brunner, N. Carqueville, D. Plencner, Orbifolds and topological defects, Commun. Math. Phys. 332 (2014) 669, arXiv:1307.3141 [hep-th]

[4] I. Brunner, N. Carqueville, D. Plencner, Discrete torsion defects, Commun. Math. Phys. 337 (2015) 429, arXiv:1404.7497 [hep-th]

[5] I. Brunner, M. Herbst, W. Lerche, B. Scheuner, Landau-Ginzburg realization of open string TFT, J. High Energy Phys. 11 (2006) 043, arXiv:hep-th/0305133

[6] I. Brunner, D. Roggenkamp, B-type defects in Landau-Ginzburg models, J. High Energy Phys. 08 (2007) 093, arXiv:0707.0922 [hep-th]

[7] R.O. Buchweitz, Maximal Cohen-Macaulay modules and Tate-cohomology over Gorenstein rings, 1986; unpublished typoscript, available from https://tspace.library.utoronto.ca/handle/1807/16682

[8] N. Carqueville, Lecture notes on 2-dimensional defect TQFT, arXiv:1607.05747 [math.QA]

[9] N. Carqueville, L. Dowdy, A. Recknagel, Algorithmic deformation of matrix factorisations, J. High Energy Phys. 04 (2012) 014, arXiv:1112.3352 [hep-th]

[10] N. Carqueville, D. Murfet, Computing Khovanov-Rozansky homology and defect fusion, Algebr. Geom. Topol. 14 (2014) 489, arXiv:1108.1081 [math.QA]

[11] N. Carqueville, D. Murfet, Adjunctions and defects in Landau-Ginzburg models, Adv. Math. 289 (2016) 480, arXiv:1208.1481 [math.AG]

[12] N. Carqueville, D. Murfet, A toolkit for defect computations in Landau-Ginzburg models, Proc. Symp. Pure Math. 90 (2015) 239, arXiv:1303.1389 [hep-th]

[13] N. Carqueville, A. Ros Camacho, I. Runkel, Orbifold equivalent potentials, J. Pure Appl. Algebra 220 (2016) 759, arXiv:1311.3354 [math.QA].

[14] N. Carqueville, I. Runkel, Orbifold completion of defect bicategories, Quantum Topol. 7 (2016) 203, arXiv:1210.6363 [math.QA]

[15] S. Cecotti, M. Del Zotto, On Arnold's 14 'Exceptional' $N=2$ superconformal gauge theories, J. High Energy Phys. 10 (2011) 099 arXiv:1107.5747 [hep-th] 
[16] M. Del Zotto, More Arnold's $N=2$ superconformal gauge theories, J. High Energy Phys. 11 (2011) 115, arXiv:1110.3826 [hep-th]

[17] T. Dyckerhoff, D. Murfet, The Kapustin-Li formula revisited, Adv. Math. 231 (2012) 1858, arXiv:1004.0687 [math.AC]

[18] T. Dyckerhoff, D. Murfet, Pushing forward matrix factorisations, Duke Math. J. 162 (2013) 1249, arXiv:1102.2957 [math.AG]

[19] D. Eisenbud, Homological algebra with an application to group representations, Trans. Amer. Math. Soc. 260 (1980) 35

[20] J. Fröhlich, J. Fuchs, I. Runkel, C. Schweigert, Defect lines, dualities, and generalised orbifolds, Proceedings of the XVI International Congress on Mathematical Physics, Prague, 2009, [arXiv:0909.5013] (math-ph)

[21] P. Griffiths, J. Harris, Principles of Algebraic Geometry, Wiley 1978

[22] M. Herbst, C.I. Lazaroiu, Localization and traces in open-closed topological LandauGinzburg models, J. High Energy Phys. 05 (2005) 044, arXiv:hep-th/0404184

[23] C. Hertling, R. Kurbel, On the classification of quasihomogeneous singularities, J. of Singularities 4 (2012) 131, arXiv:1009.0763 [math.AG]

[24] A. Kapustin, Y. Li, D-branes in Landau-Ginzburg models and algebraic geometry, J. High Energy Phys. 05 (2003) 44, arXiv:hep-th/0210296

[25] A. Kapustin, Y. Li, Topological Correlators in Landau-Ginzburg Models with Boundaries, Adv. Theor. Math. Phys. 7 (2004) 727, arXiv:hep-th/0305136

[26] A. Kapustin, Y. Li, D-branes in topological minimal models: the Landau-Ginzburg Approach, J. High Energy Phys. 07 (2004) 45, arXiv:hep-th/0306001

[27] M. Khovanov, L. Rozansky, Matrix factorizations and link homology, Fund. Math. 199 (2008) 1, arXiv:math/0401268 [math.QA]

[28] M. Krawitz, FJRW rings and Landau-Ginzburg mirror symmetry, arXiv:0906.0796 [math.AG]

[29] M. Kreuzer, H. Skarke, On the classification of quasihomogeneous functions, Commun. Math. Phys. 150 (1992) 137, arXiv:hep-th/9202039

[30] W. Lerche, C. Vafa, N.P. Warner, Chiral rings in N=2 superconformal theories, Nucl. Phys. B324 (1989) 427

[31] D. Murfet, Residues and duality for singularity categories of isolated Gorenstein singularities, Compositio Mathematica 149 (2013) 2071, arXiv:0912.1629 [math.AC]

[32] D. Murfet, The cut operation on matrix factorisations, J. Pure Appl. Algebra, in press, arXiv:1402.4541 [math.AC] 
[33] R. Newton, A. Ros Camacho, Strangely dual orbifold equivalence I, J. of Singularities 14 (2016) 34, arXiv:1509.08069 [math.QA]

[34] R. Newton, A. Ros Camacho, Orbifold autoequivalent exceptional unimodal singularities, arXiv:1607.07081 [math.QA]

[35] D. Orlov, Triangulated categories of singularities and D-branes in Landau-Ginzburg models, arXiv:math/0302304 [math.AG]

[36] A. Recknagel, P. Weinreb, Singular code to verify the statements in Thm. 4.1, and an "oeq-catalogue" with lists of orbifold equivalent potentials, based on individual examples and general properties such as Thm. 2.2 (c), https://nms.kcl.ac.uk/andreas.recknagel/oeq-page/

[37] A. Ros Camacho, Matrix factorizations and the Landau-Ginzburg/conformal field theory correspondence, arXiv:1507.06494 [math.QA]

[38] K. Saito, Duality of regular systems of weights: a précis, in: Topological Field Theory, Primitive Forms and Related Topics, M. Kashiwara, A. Matsuo, K. Saito, I. Satake (eds.), Springer 1998

[39] C. Vafa, Topological Landau-Ginzburg models, Mod. Phys. Lett. A6 (1991) 337 